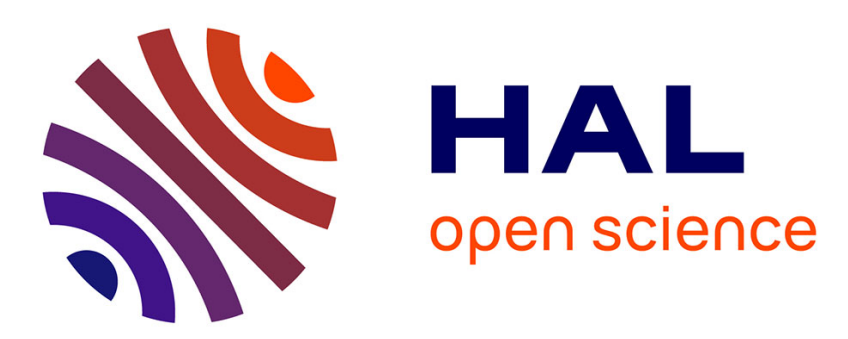

\title{
The extended finite element method combined with a modal synthesis approach for vibro-acoustic problems
}

Antoine Legay

\section{To cite this version:}

Antoine Legay. The extended finite element method combined with a modal synthesis approach for vibro-acoustic problems. International Journal for Numerical Methods in Engineering, 2014, 101, pp.329 - 350. 10.1002/nme.4798 . hal-01422329

\section{HAL Id: hal-01422329 \\ https://hal.science/hal-01422329}

Submitted on 25 Dec 2016

HAL is a multi-disciplinary open access archive for the deposit and dissemination of scientific research documents, whether they are published or not. The documents may come from teaching and research institutions in France or abroad, or from public or private research centers.
L'archive ouverte pluridisciplinaire HAL, est destinée au dépôt et à la diffusion de documents scientifiques de niveau recherche, publiés ou non, émanant des établissements d'enseignement et de recherche français ou étrangers, des laboratoires publics ou privés. 


\title{
The extended finite element method combined with a modal synthesis approach for vibro-acoustic problems
}

\author{
Antoine Legay \\ Structural $[$ Mechanics and Coupled Systems Laboratory, Conservatoire National des Arts et \Métiers, 292 rue $\square$
}

Saint-Martin, 75141 Cedex 03 Paris, France

The optimization of a vibro-acoustic problem is of main importance for passengers' comfort in transportation vehicles in terms of interior noise. Engineers use numerical tools to predict the response of this coupled problem, but it may lead to a prohibitive computational time. Based on FEM, this work aims at reducing the computational time. The first idea is to keep the same mesh of the acoustic cavity for all the structure configurations and to enrich the pressure approximation by using the extended FEM (XFEM). The enrichment is based on a Heaviside function completed at the structure tip by a continuous ramp function. The second idea is to build reduced basis. The structure basis is composed of its eigenmodes, whereas a modal synthesis method with a fixed interface is used to build the fluid basis. The interface DOFs are the enriched DOF of the XFEM, whereas the internal domain corresponds to the acoustic cavity with no structure. These two combined ideas enable to minimize the computational time in the study of the influence of the structure positions in an acoustic cavity. The method is implemented for shell structures embedded in a 3D acoustic domain.

KEY WORDS: XFEM; modal synthesis method; reduced order model; vibro-acoustics

\section{INTRODUCTION}

Noise reduction of passenger acoustic compartment in transport industry becomes a more and more important factor to be taken into account during the design process [1]. It is shown in [2] that flexible and/or absorbing structures in an acoustic cavity may have significant influences on both the amplitude of the sound pressure level and the resonant frequency shifts. These flexible and absorbing structures have to be included in the model in order to predict a realistic noise mapping in the cavity. The acoustic characteristics of such a coupled problem can be predicted using numerical tools: for instance, the FEM for the acoustic part as well as for the structural part. Engineers can study several configurations of the equipments in the passenger acoustic compartment, and they can optimize the proportion and the localization of absorbing materials in order to reach the expected noise level: the total computational time increases drastically, which may become impossible to perform in practice. In order to catch the complexity of the 3D geometry of both the acoustic domain and flexible structures, the meshes may be very fine, involving a large number of DOFS.

Noise reduction is performed in industrial applications by using additional treatments such as porous material on the walls [3] or viscoelastic material on (or inside) the structure [4]. The computational time may be reduced by the use of modal basis [5-7], subdomain decomposition [8-11], or simplification of the absorbing material as rheological devices [12-14]. Moreover, the accuracy of 
the numerical solutions may be controlled to ensure the quality of the results $[15,16]$. However, all the numerical strategies developed to reduce the computational time need a remeshing step when a new configuration of the structures in the cavity is studied.

In this work, the acoustic cavity is considered with no variation in the overall optimization process. Because the flexible structures are generally thin compared with the size of the cavity, the structures are modeled as shells. For simplicity reasons, the dissipation is only introduced in this work as a modal structural damping. These structures are localized arbitrarily in the acoustic cavity, which enables to study several configurations (different number of structures, change of the size, and the positions of the structures) with no fluid remeshing. In order to catch the pressure discontinuity around the structures in the context of incompatible meshes, the extended FEM (XFEM [17]) is used to enrich the acoustic pressure field around the structure. Because the structures are considered as shells, the normal to the structure, and so the pressure, is not defined at the structure tip: it varies continuously from one side of the structure to the other one. This effect is taken into account in the enrichment function while it is harder to model with a compatible mesh.

Based on this approach, a reduction of the structural part using a standard modal basis is proposed. Concerning the fluid part, the reduction approach is based on a modal synthesis method [8]. The XFEM discretization of the fluid part provides a natural separation between the internal and the interface DOFS: the interface DOFS are the enriched additional unknowns, whereas the internal DOFS correspond to the cavity with no structure inside. The advantage is that the modal basis of the fluid part is the same for all the configurations and then it can be computed only once. This modal basis is enriched by static modes that correspond to the response of the fluid to unit pressure imposed successively to the interface DOFS. These static modes have to be computed for each configuration. The size of the problem is finally reduced to the number of enriched fluid nodes plus the number of both structure and fluid modes, leading to a significant reduction of the number of DOFS.

The second section describes the problem and the discretized spaces and finally gives the system of equations to be solved. The details of the XFEM formulation and implementation of the structural-acoustic coupled problem can be found in [18]. The third section details the modal reduced structure basis. The fourth section gives the key idea of this work which is the original use of the XFEM DOFS separation in order to condense the fluid domain on the interface. The last section explores academic and industrial-like applications so that the convergence and the computational time reduction is shown. Finally, an influence study of the positions of 2 structures in an acoustic cavity is performed.

\section{DISCRETIZATION OF THE COUPLED PROBLEM USING XFEM}

\subsection{Description of the problem}

The acoustic fluid domain and the thin flexible structures are, respectively, denoted by $\Omega_{\mathrm{F}}$ and $\Omega_{\mathrm{S}}$ (Figure 1). The problem is studied in the frequency domain for a permanent harmonic response at angular frequency $\omega$. The fluid is assumed to be compressible and inviscid, it satisfies the classical Helmholtz equation, the pressure fluctuation is used as variable. It is characterized by the constant speed of sound $c_{0}$ and by the density $\rho_{F}$. The structural domain is assumed satisfying the elastodynamic linearized equation, the displacement field is used as variable.

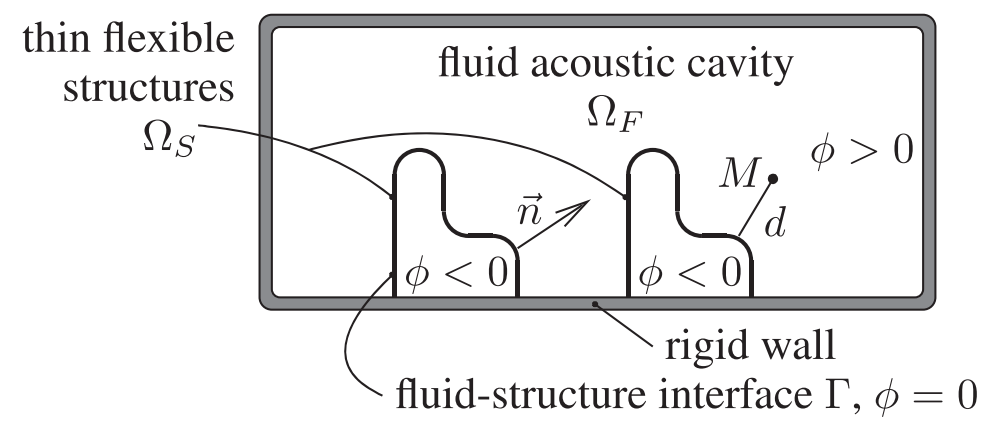

Figure 1. Description and notations of the vibro-acoustic problem. 


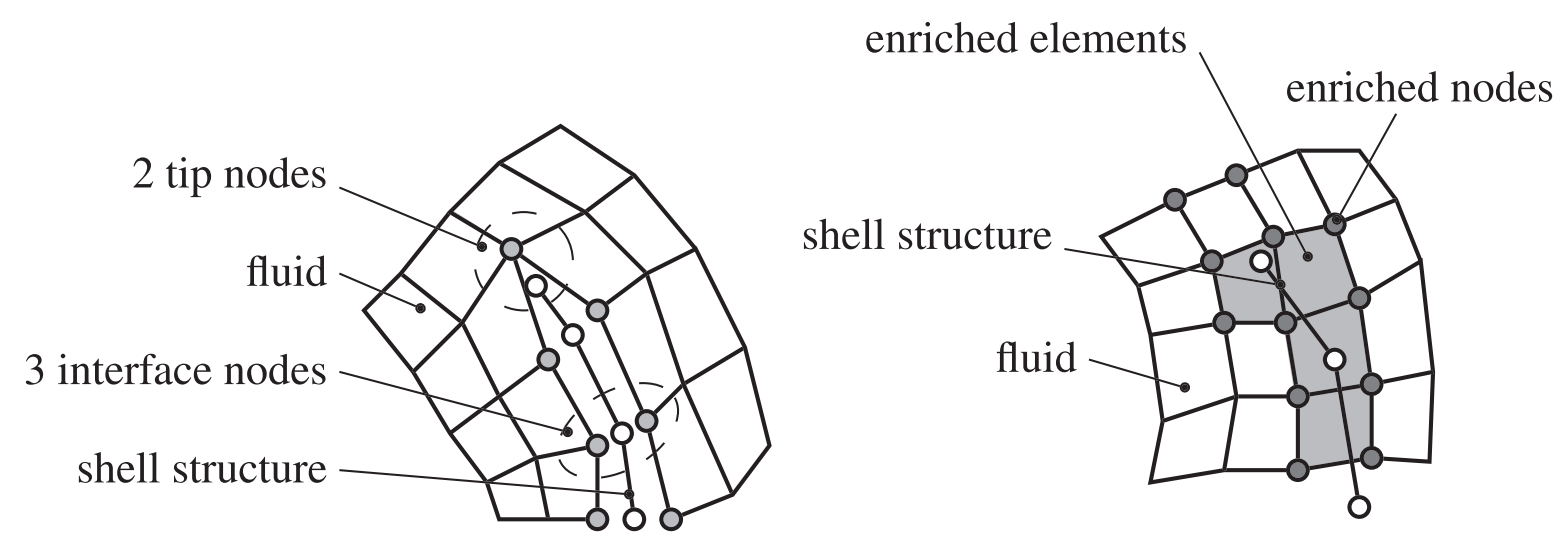

(a) classical compatible mesh

(b) XFEM non compatible mesh

Figure 2. Classical approach and XFEM approach.

The thin structures are modeled as shell structures meaning that they are seen by the fluid as surfaces. These surfaces are described in the fluid domain by the zero contour of a level set $\phi(M)$, which is the signed distance to the closest structure (Figure 1). By denoting $d$ the distance of a point $M$ in the fluid domain to the structure, the level set is defined as

$$
\phi(M)= \pm d,
$$

where the sign is determined using a conventional unit normal vector $\vec{n}$ pointing outward of one side of the structure to the arbitrarily chosen positive domain.

\subsection{Discretized fluid pressure fluctuation and structural displacement fields}

2.2.1. Fluid pressure fluctuation discretized space. A thin structure, modeled as a shell, placed in an acoustic domain separates locally the fluid into two parts, meaning that the pressure is discontinuous from one side of the structure to the other one. Moreover, the real structure tip is curve and the pressure field is continuous from one side to the other at the structure tip (Figure 3(a)). The classical discretization strategy is to make the different meshes compatible at the interface (Figure 2(a)) where three nodes are localized at the same geometrical point: two nodes for the two fluid domains and one node for the structure. The classical discretization, having one fluid node and one structure node at the same place, cannot represent correctly the local pressure field at the structure tip: in this case, the pressure has a unique value at the structure tip while it should vary. Another drawback of the classical discretization is that a remeshing is needed for each position of the structure in the fluid domain.

The alternative strategy used in this work is to embed the structure arbitrarily in the fluid mesh of the empty cavity (Figure 2(b)). The XFEM has been used in the context of fluid-structure interaction for structures embedded in a fluid flow [19-21] and for moving interface problems [22, 23], whereas it was first devoted to structural mechanics $[24,25]$. Other techniques such as immersed element method-like are developed to deal with non-compatible meshes for a fluid-structure interaction problem [26-30].

In order to correctly catch the pressure jump from one side of the structure to the other, the pressure field approximation is enriched by a Heaviside function using a partition of unity strategy [31], namely, XFEM [17]. The Heaviside function is completed at the structure tip by a continuous ramp function (Figure 3(c)). The pressure approximation becomes

$$
p(M)=\sum_{i \in \mathcal{F}} N_{\mathrm{F}}^{i}(M) P_{i}+\sum_{i \in \mathcal{A}} \psi(M) N_{\mathrm{F}}^{i}(M) A_{i},
$$

where $\mathcal{F}$ is the set of nodes of the whole fluid mesh, $N_{F}^{i}(M)$ is the standard shape function associated to the fluid node $i, P_{i}$ is the standard nodal pressure value, $\mathcal{A}$ is the set of enriched nodes, 


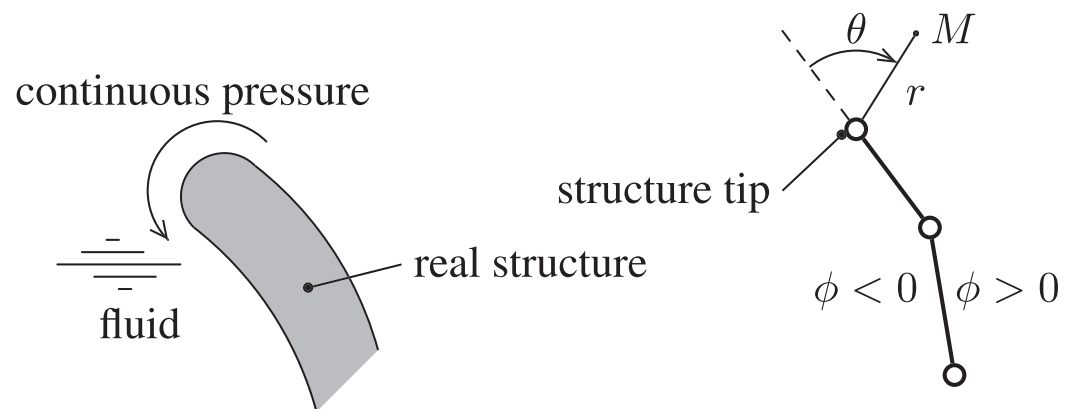

(a) real structure tip

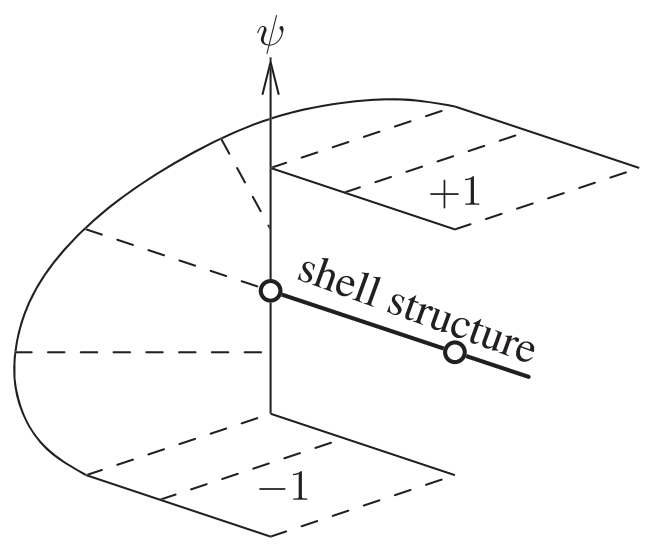

(c) enrichment function

Figure 3. XFEM approximation at the interface.

$\psi(M)$ is the enrichment function, and $A_{i}$ is the additional nodal unknown coming from the enrichment. The set of enriched nodes are those that are connected to the elements cut by the structures (Figure 2(b)). The enrichment function $\psi$ is defined by

$$
\begin{aligned}
& \psi(M)=\operatorname{sign}(\phi(M)) \text { if }|\theta(M)| \geqslant \frac{\pi}{2}, \\
& \psi(M)=\frac{2}{\pi} \theta(M) \text { if }|\theta(M)|<\frac{\pi}{2},
\end{aligned}
$$

where $\theta$ is defined in Figure 3(b). $\theta$ has the same sign as the level set, it is equal to zero in the continuation of the structure. This enrichment function enables to have a continuous variation of pressure at the structure tip because $-1 \leqslant \psi \leqslant+1$ if $r=0$.

For tridimensional applications, the structure edge is curve and the $\theta$ angle is measured in the local normal plane to this curve. In this work, the structure edge is supposed to be smooth enough such that it does not contain corners.

The full approximation containing both the standard part and the enrichment can be written from Equation (2) as

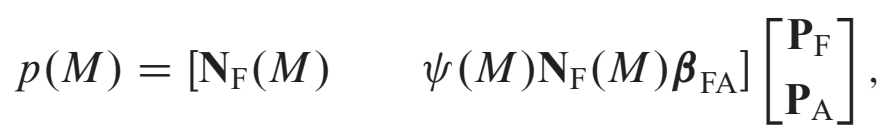

where $\mathbf{N}_{\mathrm{F}}$ is the shape function matrix of the fluid, $\mathbf{P}_{\mathrm{F}}$ contains the nodal pressure of the standard part, and $\mathbf{P}_{\mathrm{A}}$ contains the nodal additional unknowns coming from the enrichment. The Boolean localization matrix $\boldsymbol{\beta}_{F A}$ is used to extract enriched set of nodes $\mathcal{A}$ from the set of nodes $\mathcal{F}$.

The tridimensional acoustic domain is discretized by four-node tetrahedral elements in this work. There are blending elements in the discretization, these are the elements that are connected to both enriched and non-enriched nodes [32]. These elements have spurious effects only for the elements close to the structure tip enrichment. For the others, the Heaviside enrichment does not introduce errors in the solution. Because these spurious effects affect only a few elements, no correction has been implemented and no significant error has been found in the various numerical tests.

2.2.2. Structural displacement discretized space. The thin structures are discretized using shell elements, three-node discrete kirchhoff triangle (DKT) in this work (Kirchhoff-Love theory) [33-36]. The structure DOFS are collected in $\mathbf{U}_{\mathrm{S}}$, which is linked to the structural displacement by

$$
\mathbf{u}_{\mathrm{S}}=\mathbf{N}_{\mathrm{S}} \mathbf{U}_{\mathrm{S}},
$$

where $\mathbf{N}_{\mathrm{S}}$ is the shape function matrix. The stiffness and mass matrices of the structure are, respectively, denoted by $\mathbf{K}_{\mathrm{SS}}$ and $\mathbf{M}_{\mathrm{SS}}$. A structural damping matrix $\mathbf{D}_{\mathrm{SS}}$ is introduced in the following using a modal damping. 


\subsection{Discretized weak form of the coupled problem}

The continuous weak form of the equations of the structural-acoustic coupled problem at a given angular frequency $\omega \neq 0$ gives a linear system of equations in the discretized fluid and structure spaces. Because a structural damping is considered, the solution is complex and the imaginary unit number $j$ is introduced $\left(j^{2}=-1\right)$. The following system is obtained:

$$
\left(\left[\begin{array}{ccc}
\mathbf{K}_{\mathrm{FF}} & \mathbf{K}_{\mathrm{FA}} & \mathbf{0} \\
\mathbf{K}_{\mathrm{FA}}^{T} & \mathbf{K}_{\mathrm{AA}} & \mathbf{0} \\
\mathbf{0} & -\mathbf{C}_{\mathrm{AS}}^{T} & \mathbf{K}_{\mathrm{SS}}+\omega j \mathbf{D}_{\mathrm{SS}}
\end{array}\right]-\omega^{2}\left[\begin{array}{ccc}
\mathbf{M}_{\mathrm{FF}} & \mathbf{M}_{\mathrm{FA}} & \mathbf{0} \\
\mathbf{M}_{\mathrm{FA}}^{T} & \mathbf{M}_{\mathrm{AA}} & \mathbf{C}_{\mathrm{AS}} \\
\mathbf{0} & \mathbf{0} & \mathbf{M}_{\mathrm{SS}}
\end{array}\right]\right)\left[\begin{array}{c}
\mathbf{P}_{\mathrm{F}} \\
\mathbf{P}_{\mathrm{A}} \\
\mathbf{U}_{\mathrm{S}}
\end{array}\right]=\left[\begin{array}{c}
\omega^{2} \mathbf{U}_{\mathrm{F}} \\
\mathbf{0} \\
\mathbf{F}_{\mathrm{S}}
\end{array}\right],
$$

where $\mathbf{F}_{\mathrm{S}}$ is the discretized external load on the structure, $\mathbf{U}_{\mathrm{F}}$ is the discretized particle displacement, and the other matrices are defined as

$$
\begin{aligned}
\mathbf{K}_{\mathrm{FF}} & =\frac{1}{\rho_{f}} \int_{\Omega_{F}} \mathbf{B}_{\mathrm{F}}^{T} \mathbf{B}_{\mathrm{F}} \mathrm{d} V, \\
\mathbf{M}_{\mathrm{FF}} & =\frac{1}{\rho_{f} c_{0}^{2}} \int_{\Omega_{F}} \mathbf{N}_{\mathrm{F}}^{T} \mathbf{N}_{\mathrm{F}} \mathrm{d} V, \\
\mathbf{K}_{\mathrm{AA}} & =\boldsymbol{\beta}_{\mathrm{FA}}^{T} \frac{1}{\rho_{f}} \int_{\Omega_{F}} \mathbf{B}_{\mathrm{A}}^{T} \mathbf{B}_{\mathrm{A}} \mathrm{d} V \boldsymbol{\beta}_{\mathrm{FA}}, \\
\mathbf{M}_{\mathrm{AA}} & =\boldsymbol{\beta}_{\mathrm{FA}}^{T} \frac{1}{\rho_{f} c_{0}^{2}} \int_{\Omega_{F}} \psi^{2} \mathbf{N}_{\mathrm{F}}^{T} \mathbf{N}_{\mathrm{F}} \mathrm{d} V \boldsymbol{\beta}_{\mathrm{FA}}, \\
\mathbf{K}_{\mathrm{FA}} & =\frac{1}{\rho_{f}} \int_{\Omega_{F}} \mathbf{B}_{\mathrm{F}}^{T} \mathbf{B}_{\mathrm{A}} \mathrm{d} V \boldsymbol{\beta}_{\mathrm{FA}}, \\
\mathbf{M}_{\mathrm{FA}} & =\frac{1}{\rho_{f} c_{0}^{2}} \int_{\Omega_{F}} \psi \mathbf{N}_{\mathrm{F}}^{T} \mathbf{N}_{\mathrm{F}} \mathrm{d} V \boldsymbol{\beta}_{\mathrm{FA}}, \\
\mathbf{C}_{\mathrm{AS}} & =2 \boldsymbol{\beta}_{\mathrm{FA}} \int_{\Gamma} \mathbf{N}_{\mathrm{F}}^{T} \mathbf{n}^{T} \mathbf{N}_{\mathrm{S}} \mathrm{d} \Sigma, \\
\text { with } \mathbf{B}_{\mathrm{F}}= & {\left[\frac{\partial \mathbf{N}_{\mathrm{F}}^{T}}{\partial x} \frac{\partial \mathbf{N}_{\mathrm{F}}^{T}}{\partial y} \frac{\partial \mathbf{N}_{\mathrm{F}}^{T}}{\partial z}\right]^{T}, } \\
\text { and } \mathbf{B}_{\mathrm{A}}= & {\left[\psi \frac{\partial \mathbf{N}_{\mathrm{F}}^{T}}{\partial x}+\frac{\partial \psi}{\partial x} \mathbf{N}_{\mathrm{F}}^{T}\left|\psi \frac{\partial \mathbf{N}_{\mathrm{F}}^{T}}{\partial y}+\frac{\partial \psi}{\partial y} \mathbf{N}_{\mathrm{F}}^{T}\right| \psi \frac{\partial \mathbf{N}_{\mathrm{F}}^{T}}{\partial z}+\frac{\partial \psi}{\partial z} \mathbf{N}_{\mathrm{F}}^{T}\right]^{T} . }
\end{aligned}
$$

The coupling between the acoustic fluid and the structures is only realized between the structural displacement $\mathbf{U}_{\mathrm{S}}$ and the pressure enrichment $\mathbf{P}_{\mathrm{A}}$ through the coupling matrix $\mathbf{C}_{\mathrm{AS}}$. The details of the numerical implementations of the aforementioned matrices are given in [18].

\section{MODAL REDUCTION OF THE STRUCTURE}

The structure reduced basis is chosen as the modal basis is enriched with the static solution. Each vector $\boldsymbol{\phi}_{\mathrm{S}}^{i}$ of the modal basis $\boldsymbol{\Phi}_{\mathrm{Sm}}$, composed of the $m$ first eigenmodes, is the solution of the following structural eigenvalue problem:

$$
\left(\mathbf{K}_{\mathrm{SS}}-\omega_{\mathrm{S}}^{i^{2}} \mathbf{M}_{\mathrm{SS}}\right) \boldsymbol{\phi}_{\mathrm{S}}^{i}=\mathbf{0},
$$

where the boundary conditions on the structure are taken into account. The modes are normalized according to the mass matrix 


$$
\boldsymbol{\Phi}_{\mathrm{Sm}}^{T} \mathbf{M}_{\mathrm{SS}} \boldsymbol{\Phi}_{\mathrm{Sm}}=\mathbf{1}_{\mathrm{mm}},
$$

and the diagonal generalized stiffness matrix $\boldsymbol{\Omega}_{\mathrm{mm}}$ is obtained:

$$
\boldsymbol{\Omega}_{\mathrm{mm}}=\boldsymbol{\Phi}_{\mathrm{Sm}}{ }^{T} \mathbf{K}_{\mathrm{SS}} \boldsymbol{\Phi}_{\mathrm{Sm}} .
$$

The static solution $\boldsymbol{\Psi}_{S}$ is the solution of the following system of equations:

$$
\mathbf{K}_{\mathrm{SS}} \boldsymbol{\Psi}_{\mathrm{S}}=\mathbf{F}_{\mathrm{S}},
$$

where $\boldsymbol{\Psi}_{\mathrm{S}}$ satisfies the boundary conditions. In the case of a singular stiffness matrix, an arbitrary shift using the mass matrix can be introduced. This vector is made orthogonal to the modal basis $\boldsymbol{\Phi}_{\text {Sm }}$ by using a Gram-Schmidt procedure. It is finally normalized according to the mass matrix

$$
\boldsymbol{\Psi}_{\mathrm{S}}^{T} \mathbf{M}_{\mathrm{SS}} \boldsymbol{\Psi}_{\mathrm{S}}=1
$$

Using the combination of the modal basis and the static mode, the structural reduced basis is denoted by $\boldsymbol{\Phi}_{\mathrm{Sn}}$

$$
\boldsymbol{\Phi}_{\mathrm{Sn}}=\left[\boldsymbol{\Phi}_{\mathrm{Sm}} \boldsymbol{\Psi}_{S}\right],
$$

where $n=m+1$ is the number of columns of $\boldsymbol{\Phi}_{\mathrm{Sn}}$. The structural displacement vector expressed in the reduced basis $\boldsymbol{\Phi}_{\mathrm{Sn}}$ is denoted by $\boldsymbol{\alpha}_{\mathrm{n}}$. The projected stiffness matrix of the structure in this reduced basis is

$$
\mathbf{K}_{\mathrm{nn}}=\left[\begin{array}{cc}
\boldsymbol{\Omega}_{\mathrm{mm}} & \mathbf{0}_{\mathrm{m}} \\
\mathbf{0}_{\mathrm{m}}^{T} & \boldsymbol{\Psi}_{\mathrm{S}}^{T} \mathbf{K}_{\mathrm{SS}} \boldsymbol{\Psi}_{\mathrm{S}}
\end{array}\right],
$$

where $\mathbf{0}_{\mathrm{m}}$ is a column of zeros of size $m$.

Because a modal damping is introduced, the projected structural damping matrix $\mathbf{D}_{\mathrm{nn}}$ is diagonal. Its $i^{\text {th }}$ term corresponds to the modal damping $2 \xi_{i} \omega_{\mathrm{S}}^{i}$, where $\xi_{i}$ is the modal damping factor.

The coupling system of Equation (6) becomes

$$
\left(\left[\begin{array}{ccc}
\mathbf{K}_{\mathrm{FF}} & \mathbf{K}_{\mathrm{FA}} & \mathbf{0} \\
\mathbf{K}_{\mathrm{FA}}^{T} & \mathbf{K}_{\mathrm{AA}} & \mathbf{0} \\
\mathbf{0} & -\mathbf{C}_{\mathrm{An}}^{T} & \mathbf{K}_{\mathrm{nn}}+\omega j \mathbf{D}_{\mathrm{nn}}
\end{array}\right]-\omega^{2}\left[\begin{array}{ccc}
\mathbf{M}_{\mathrm{FF}} & \mathbf{M}_{\mathrm{FA}} & \mathbf{0} \\
\mathbf{M}_{\mathrm{FA}}^{T} & \mathbf{M}_{\mathrm{AA}} & \mathbf{C}_{\mathrm{An}} \\
\mathbf{0} & \mathbf{0} & \mathbf{1}_{\mathrm{nn}}
\end{array}\right]\right)\left[\begin{array}{c}
\mathbf{P}_{\mathrm{F}} \\
\mathbf{P}_{\mathrm{A}} \\
\boldsymbol{\alpha}_{\mathrm{n}}
\end{array}\right]=\left[\begin{array}{c}
\omega^{2} \mathbf{U}_{\mathrm{F}} \\
\mathbf{0} \\
\mathbf{F}_{\mathrm{n}}
\end{array}\right],
$$

where $\mathbf{1}_{\text {nn }}$ is the identity matrix of size $n$. The coupling matrix between the acoustic fluid and the pressure enrichment is defined by

$$
\mathbf{C}_{\mathrm{An}}=\mathbf{C}_{\mathrm{AS}} \boldsymbol{\Phi}_{\mathrm{Sn}},
$$

and the generalized structural force is given by

$$
\mathbf{F}_{\mathrm{n}}=\boldsymbol{\Phi}_{\mathrm{Sn}}^{T} \mathbf{F}_{\mathrm{S}} .
$$

\section{MODAL SYNTHESIS REDUCTION OF THE ACOUSTIC DOMAIN}

A restrained-interface component mode synthesis method is chosen in order to condense the acoustic domain on the fluid-structure interface following the classical strategy used in structural mechanics, known as Craig-Bampton method [37]. This strategy is applied in [8] for a structural-acoustic problem using compatible meshes between the fluid and the structures, it is shown that the computational time is significantly reduced. 


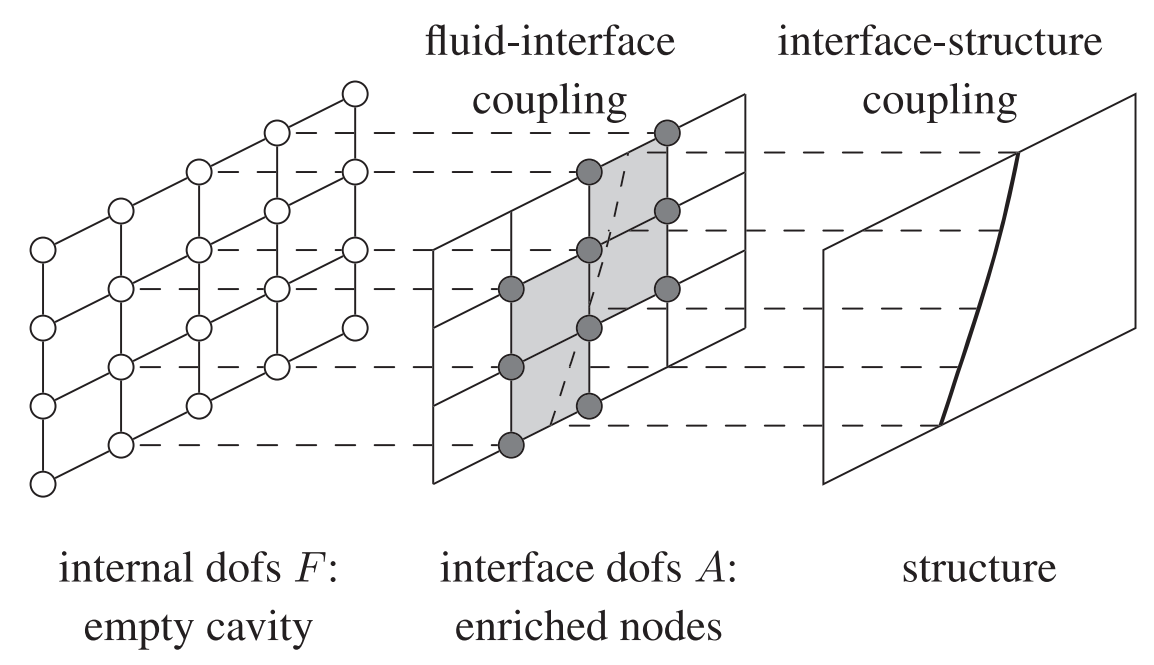

Figure 4. Separation of the DOFS into internal $(F)$ and interface $(A)$.

The structural-acoustic problem written as the system of Equation (15) provides a natural separation between the fluid DOFS coupled to the structure (interface DOFS) and the uncoupled ones (internal DOFS): in the present work, the interface DOFS are those denoted by the subscript A, whereas the internal DOFS are those denoted by the subscript F (Figure 4). An acoustic reduction basis of the internal DOFS is built using a truncated low frequency modal basis. It is completed by static modes taking into account mobility of the interface DOFS, contribution of higher order modes to the response, and separations of the acoustic cavity by the structures. Static modes are computed with pressure DOFS 'restrained' at interface $\left(\mathbf{P}_{\mathrm{A}}=\mathbf{0}\right)$ and are solution of the following eigenvalue problem

$$
\left(\mathbf{K}_{\mathrm{FF}}-\omega_{\mathrm{F}}^{i^{2}} \mathbf{M}_{\mathrm{FF}}\right) \boldsymbol{\phi}_{\mathrm{F}}^{i}=\mathbf{0} .
$$

The $p$ first modes are collected in the $\boldsymbol{\Phi}_{\mathrm{Fp}}$ matrix. The modes are normalized according to the mass matrix

$$
\boldsymbol{\Phi}_{\mathrm{Fp}}^{T} \mathbf{M}_{\mathrm{FF}} \boldsymbol{\Phi}_{\mathrm{Fp}}=\mathbf{1}_{\mathrm{pp}}
$$

and the diagonal generalized stiffness matrix $\boldsymbol{\Omega}_{\mathrm{pp}}$ is obtained,

$$
\boldsymbol{\Omega}_{\mathrm{pp}}=\boldsymbol{\Phi}_{\mathrm{Fp}}^{T} \mathbf{K}_{\mathrm{FF}} \boldsymbol{\Phi}_{\mathrm{Fp}}
$$

It is noteworthy that the internal DOFS are the ones corresponding to the empty acoustic cavity with no immersed structure inside.

The interface static modes are computed as successive responses of the empty cavity to a unit prescribed enriched pressure value. For the $\mathrm{i}^{t h}$ enriched node, the corresponding static mode $\boldsymbol{\Psi}_{\mathrm{F}}^{i}$ is the solution of the following system

$$
\left[\begin{array}{ll}
\mathbf{K}_{\mathrm{FF}} & \mathbf{K}_{\mathrm{FA}}
\end{array}\right]\left[\begin{array}{c}
\boldsymbol{\Psi}_{\mathrm{F}}^{i} \\
\mathbf{1}_{\mathrm{A}}^{i}
\end{array}\right]=\left[\begin{array}{l}
\mathbf{0}_{\mathrm{F}}
\end{array}\right],
$$

where $\mathbf{1}_{\mathrm{A}}^{i}$ denotes a column vector of 0 with 1 located at the $\mathrm{i}^{\text {th }}$ row. The obtained static modes are collected in the matrix $\Psi_{\mathrm{FA}}$, which can also be written from a mathematical point of view as

$$
\Psi_{\mathrm{FA}}=-\mathbf{K}_{\mathrm{FF}}^{-1} \mathbf{K}_{\mathrm{FA}}
$$


In the case of a singular stiffness matrix, an arbitrary shift using the mass matrix can be introduced.

The discretized fluid pressure field is written using both the modal basis and the static modes as

$$
\left[\begin{array}{l}
\mathbf{P}_{\mathrm{F}} \\
\mathbf{P}_{\mathrm{A}}
\end{array}\right]=\left[\begin{array}{ll}
\boldsymbol{\Phi}_{\mathrm{Fp}} & \boldsymbol{\Psi}_{\mathrm{FA}} \\
\mathbf{0}_{\mathrm{Ap}} & \mathbf{1}_{\mathrm{AA}}
\end{array}\right]\left[\begin{array}{l}
\boldsymbol{\alpha}_{\mathrm{p}} \\
\mathbf{P}_{\mathrm{A}}
\end{array}\right]
$$

where $\left[\begin{array}{ll}\boldsymbol{\alpha}_{\mathrm{p}}^{T} & \mathbf{P}_{\mathrm{A}}^{T}\end{array}\right]^{T}$ is the pressure field expressed in the reduced basis $\boldsymbol{\Xi}$ defined as (using Equation (4) and (23))

$$
\boldsymbol{\Xi}=\left[\mathbf{N}_{\mathrm{F}}(M) \boldsymbol{\Phi}_{\mathrm{Fp}} \quad \mathbf{N}_{\mathrm{F}}(M) \boldsymbol{\Psi}_{\mathrm{FA}}+\psi(M) \mathbf{N}_{\mathrm{F}}(M) \boldsymbol{\beta}_{\mathrm{FA}}\right]
$$

The reduced coupled system of equations can be written by replacing Equation (23) into Equation (15) as

$$
\left(\left[\begin{array}{ccc}
\boldsymbol{\Omega}_{\mathrm{pp}} & \mathbf{0} & \mathbf{0} \\
\mathbf{0} & \hat{\mathbf{K}}_{\mathrm{AA}} & \mathbf{0} \\
\mathbf{0} & -\mathbf{C}_{\mathrm{An}}^{T} & \mathbf{K}_{\mathrm{nn}}+\omega j \mathbf{D}_{\mathrm{nn}}
\end{array}\right]-\omega^{2}\left[\begin{array}{ccc}
\mathbf{1}_{\mathrm{pp}} & \mathbf{M}_{\mathrm{pA}} & \mathbf{0} \\
\mathbf{M}_{\mathrm{pA}}^{T} & \hat{\mathbf{M}}_{\mathrm{AA}} & \mathbf{C}_{\mathrm{An}} \\
\mathbf{0} & \mathbf{0} & \mathbf{1}_{\mathrm{nn}}
\end{array}\right]\right)\left[\begin{array}{c}
\boldsymbol{\alpha}_{\mathrm{p}} \\
\mathbf{P}_{\mathrm{A}} \\
\boldsymbol{\alpha}_{\mathrm{n}}
\end{array}\right]=\left[\begin{array}{c}
\omega^{2} \boldsymbol{\Phi}_{\mathrm{Fp}}^{T} \mathbf{U}_{\mathrm{F}} \\
\mathbf{0} \\
\mathbf{F}_{\mathrm{n}}
\end{array}\right],
$$

where the matrices are defined by

$$
\begin{aligned}
\hat{\mathbf{K}}_{\mathrm{AA}} & =\mathbf{K}_{\mathrm{AA}}+\boldsymbol{\Psi}_{\mathrm{FA}}^{T} \mathbf{K}_{\mathrm{FA}}, \\
\hat{\mathbf{M}}_{\mathrm{AA}} & =\boldsymbol{\Psi}_{\mathrm{FA}}^{T} \stackrel{\star}{\mathbf{M}_{\mathrm{FA}}}+\mathbf{M}_{\mathrm{FA}}^{T} \boldsymbol{\Psi}_{\mathrm{FA}}+\mathbf{M}_{\mathrm{AA}}, \\
\mathbf{M}_{\mathrm{pA}} & =\boldsymbol{\Phi}_{\mathrm{Fp}}^{T} \stackrel{\star}{\mathrm{M}}_{\mathrm{FA}} \\
\stackrel{\mathbf{M}}{\mathrm{FA}} & =\mathbf{M}_{\mathrm{FF}} \boldsymbol{\Psi}_{\mathrm{FA}}+\mathbf{M}_{\mathrm{FA}} .
\end{aligned}
$$

This system of equations can be solved for each frequency step and is referred to as the noncondensed version in the following. However, the $\alpha_{\mathrm{p}}$ unknowns can be eliminated using the first line of the system

$$
\boldsymbol{\alpha}_{\mathrm{p}}=\omega^{2}\left(\boldsymbol{\Omega}_{\mathrm{pp}}-\omega^{2} \mathbf{1}_{\mathrm{pp}}\right)^{-1}\left(\boldsymbol{\Phi}_{\mathrm{Fp}}^{T} \mathbf{U}_{\mathrm{F}}+\mathbf{M}_{\mathrm{pA}} \mathbf{P}_{\mathrm{A}}\right)
$$

After replacing in the second line, the reduced system is

$$
\left(\left[\begin{array}{cc}
\hat{\mathbf{K}}_{\mathrm{AA}} & \mathbf{0} \\
-\mathbf{C}_{\mathrm{An}}^{T} & \mathbf{K}_{\mathrm{nn}}+\omega j \mathbf{D}_{\mathrm{nn}}
\end{array}\right]-\omega^{2}\left[\begin{array}{cc}
\tilde{\mathbf{M}}_{\mathrm{AA}}(\omega) & \mathbf{C}_{\mathrm{An}} \\
\mathbf{0} & \mathbf{1}_{\mathrm{nn}}
\end{array}\right]\right)\left[\begin{array}{c}
\mathbf{P}_{\mathrm{A}} \\
\boldsymbol{\alpha}_{\mathrm{n}}
\end{array}\right]=\left[\begin{array}{c}
\tilde{\mathbf{F}}_{\mathrm{A}}(\omega) \\
\mathbf{F}_{\mathrm{n}}
\end{array}\right]
$$

with

$$
\tilde{\mathbf{F}}_{\mathrm{A}}(\omega)=\omega^{4} \mathbf{M}_{\mathrm{pA}}^{T}\left(\boldsymbol{\Omega}_{\mathrm{pp}}-\omega^{2} \mathbf{1}_{\mathrm{pp}}\right)^{-1} \boldsymbol{\Phi}_{\mathrm{Fp}}^{T} \mathbf{U}_{\mathrm{F}},
$$

and

$$
\tilde{\mathbf{M}}_{\mathrm{AA}}(\omega)=\hat{\mathbf{M}}_{\mathrm{AA}}+\omega^{2} \mathbf{M}_{\mathrm{pA}}^{T}\left(\boldsymbol{\Omega}_{\mathrm{pp}}-\omega^{2} \mathbf{1}_{\mathrm{pp}}\right)^{-1} \mathbf{M}_{\mathrm{pA}}
$$


This system of equations is referred to as the condensed version in the following. After solving this system, the pressure field in the cavity can be deduced by using successively Equations (30) and (24). Note that the inverse matrix in Equation (33) is diagonal and is trivial to solve at each frequency step.

\section{PRACTICAL USE OF THE PROPOSED METHOD}

In order to clarify the practical implementation and to highlight the advantages of the presently proposed reduced approaches, the algorithms are detailed in the following. In the context of an optimization, uncertainty or reliability study of the structural-acoustic problem, several configurations have to be tested. It is supposed that the varying parameters are those related to the number of structures and to their positions. In the following, a configuration is a chosen set of parameters.

The classical approach, as used in a commercial software, has a conforming mesh at the fluidstructure interface and does not perform any reduction. As presented in the Algorithm 1, the fluid mesh is built for each configuration. The matrices associated to the fluid and to the fluidstructure coupling are computed for each configuration. The generic notations $\mathcal{K}, \mathcal{D}$, and $\mathcal{M}$ denote, respectively, the stiffness, the damping, and the mass matrices of the structural-acoustic coupled problem.

The algorithm of the proposed reduced method, in its non-condensed version, is presented in Algorithm 2. The empty cavity fluid mesh and the structure meshes are built once before the loop over the configurations. The corresponding uncoupled matrices $\mathbf{K}_{\mathrm{SS}}, \mathbf{M}_{\mathrm{SS}}, \mathbf{K}_{\mathrm{FF}}$, and $\mathbf{M}_{\mathrm{FF}}$ as well as the fluid and structure modal basis $\boldsymbol{\Phi}_{\mathrm{Sn}}$ and $\boldsymbol{\Phi}_{\mathrm{Fp}}$ are computed at this step. A geometry variation of the structures is possible but the structure matrices $\mathbf{K}_{\mathrm{SS}}, \mathbf{M}_{\mathrm{SS}}$, and $\boldsymbol{\Phi}_{\mathrm{Sn}}$ have then to be computed for each configuration. In this paper, only variations of the structure positions are considered. For each configuration, the level set $\phi(M)$ and the enriched set of nodes $\mathcal{A}$ have to be updated. This allows to perform the computation of the static modes $\boldsymbol{\Psi}_{\mathrm{FA}}$ and the coupling matrix $\mathbf{C}_{\mathrm{An}}$. The matrices $\hat{\mathbf{K}}_{\mathrm{AA}}$ and $\hat{\mathbf{M}}_{\mathrm{AA}}$ are then computed. For each frequency step, the system of Equation (25) is solved; the fluid pressure and the structure displacement are deduced using, respectively, the reduced basis $\boldsymbol{\Xi}$ and $\boldsymbol{\Phi}_{\mathrm{Sn}}$.

The algorithm of the proposed reduced method, in its condensed version, is detailed in Algorithm 3. The difference with the non-condensed version lies in the frequency loop. For each frequency, the condensed matrix $\tilde{\mathbf{M}}_{\mathrm{AA}}(\omega)$ has to be computed, involving the trivial inversion of a diagonal matrix. After solving the system of Equation (31), the modal fluid contribution $\boldsymbol{\alpha}_{\mathrm{p}}$ is computed using Equation (30). The fluid pressure and the structure displacement are computed as previously. This condensed version is very powerful when the number of interface DOFS is small compared with the number of fluid modes. However, because this is not usually the case in this work, the condensed version may be less efficient than the non-condensed version.

All the numerical developments are done within Python at the global level, coupled to Fortran routines for elemental computations.

\section{Algorithm 1 Classic approach}

- Build structure meshes

- Compute structure matrices

- Loop over the configurations

- Build compatible fluid mesh

- Compute fluid and coupling matrices

- Form the matrices $\mathcal{K}, \mathcal{D}$ and $\mathcal{M}$

- Loop over the frequency range

$$
\text { * Solve }\left(\mathcal{K}+\omega j \mathcal{D}-\omega^{2} \mathcal{M}\right) \mathcal{X}=\mathcal{F}
$$


Algorithm 2 XFEM reduced approach, no condensation

- Build structure meshes

- Build empty cavity fluid mesh

- Compute the structure and fluid matrices $\mathbf{K}_{\mathrm{SS}}, \mathbf{M}_{\mathrm{SS}}, \mathbf{K}_{\mathrm{FF}}, \mathbf{M}_{\mathrm{FF}}$

- Compute the structure modal basis $\boldsymbol{\Phi}_{\mathrm{Sn}}$

- Compute the fluid modal basis $\boldsymbol{\Phi}_{\mathrm{Fp}}$

- Loop over the configurations

- Compute the level-set $\phi(M)$

- Get set of enriched nodes $\mathcal{A}$

- Compute static modes $\boldsymbol{\Psi}_{\mathrm{FA}}$

- Compute coupling matrix $\mathbf{C}_{\mathrm{An}}$

- Compute matrices $\hat{\mathbf{K}}_{\mathrm{AA}}$ and $\hat{\mathbf{M}}_{\mathrm{AA}}$

- Loop over the frequency range

* Solve the reduced system of Eqs. (25)

* Get fluid pressure using the reduced basis $\Xi$

* Get structure displacement using the reduced basis $\boldsymbol{\Phi}_{\mathrm{Sn}}$

Algorithm 3 XFEM reduced approach, with condensation

- Build structure meshes

- Build empty cavity fluid mesh

- Compute the structure and fluid matrices $\mathbf{K}_{\mathrm{SS}}, \mathbf{M}_{\mathrm{SS}}, \mathbf{K}_{\mathrm{FF}}, \mathbf{M}_{\mathrm{FF}}$

- Compute the structure modal basis $\boldsymbol{\Phi}_{\mathrm{Sn}}$

- Compute the fluid modal basis $\boldsymbol{\Phi}_{\mathrm{Fp}}$

- Loop over the configurations

- Compute the level-set $\phi(M)$

- Get set of enriched nodes $\mathcal{A}$

- Compute static modes $\boldsymbol{\Psi}_{\mathrm{FA}}$

- Compute coupling matrix $\mathbf{C}_{\mathrm{An}}$

- Compute matrices $\hat{\mathbf{K}}_{\mathrm{AA}}$ and $\hat{\mathbf{M}}_{\mathrm{AA}}$

- Loop over the frequency range

* Compute the $\tilde{\mathbf{M}}_{\mathrm{AA}}(\omega)$ matrix

* Eventually, compute $\tilde{\mathbf{F}}_{\mathrm{A}}(\omega)$

* Solve the reduced system of Eqs. (31)

* Compute $\boldsymbol{\alpha}_{\mathrm{p}}$ using Eq. (30)

* Get fluid pressure using the reduced basis $\Xi$

* Get structure displacement using the reduced basis $\boldsymbol{\Phi}_{\mathrm{Sn}}$

\section{APPLICATIONS}

\subsection{Validation of the tip enrichment}

One of the originalities of the present work compared with the previous enrichment proposed by the author [18] is to enable free structure edges in the acoustic fluid. In order to validate this edge enrichment, a rigid wall is immersed in a thin square cavity bounded by rigid walls (Figure 5 and Table I). A harmonic unit pressure is imposed on the left side of the cavity. Boundary conditions as well as load are chosen such that the solution of the problem is two-dimensional.

In order to catch correctly the local pressure variation around the top of the structure tip, the structure has a small thickness $e$ for the classical approach using a conforming mesh. This reference solution is computed using a fine mesh consisting of 153,637 nodes and 305,311 three-node triangle 


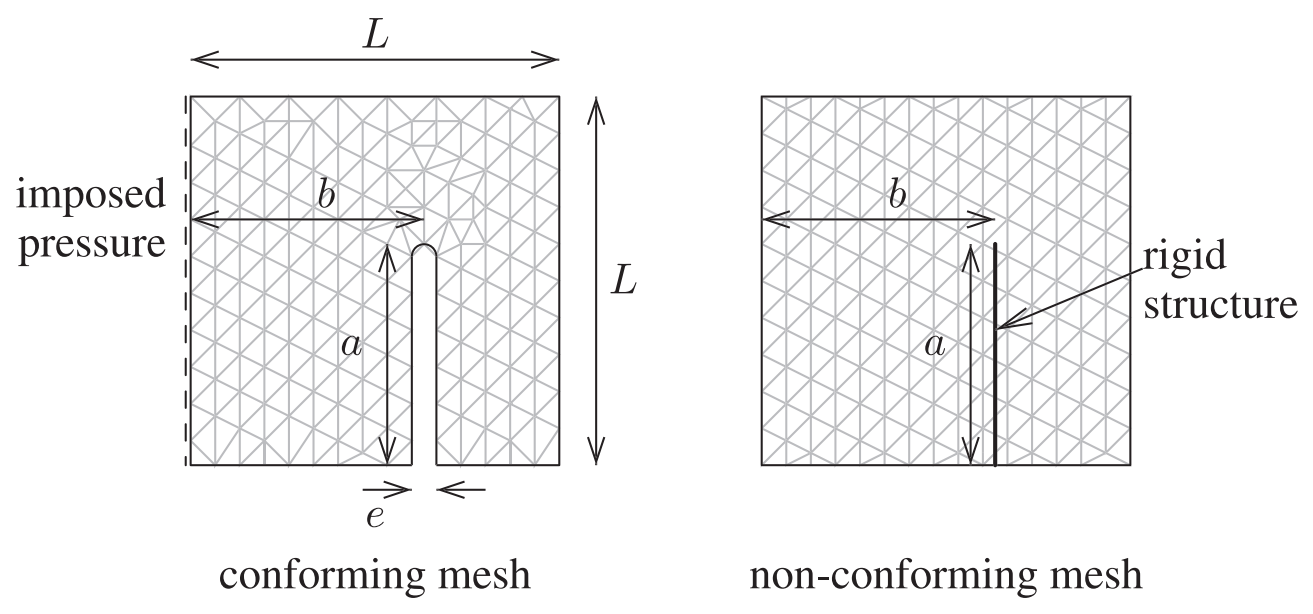

Figure 5. Geometry of the separated cavity.

Table I. Data of the separated cavity.

\begin{tabular}{ll}
\hline$L(\mathrm{~m})$ & \multicolumn{1}{c}{1} \\
$a(\mathrm{~m})$ & 0.6 \\
$b(\mathrm{~m})$ & 0.65 \\
$e(\mathrm{~m})$ & 0.0004 \\
$c_{0}\left(\mathrm{~m} \mathrm{~s}^{-1}\right)$ & 340 \\
$\rho_{F}\left(\mathrm{~kg} \mathrm{~m}^{-3}\right)$ & 1.2 \\
\hline
\end{tabular}

Table II. Different non-compatible used meshes of the separated cavity.

\begin{tabular}{ccccccc}
\hline & Mesh 1 & Mesh 2 & Mesh 3 & Mesh 4 & Mesh 5 & Mesh 6 \\
\hline nb. nodes & 13 & 123 & 963 & 8554 & 77,653 & 161,196 \\
\hline
\end{tabular}

elements. The semicircle structure tip is discretized by 47 nodes, which give an accurate solution of the local pressure at this point.

The convergence study of the proposed method using a tip enrichment is realized using six nonconforming meshes from meshes 1 to 6 (Table II). These meshes are unstructured and have a uniform element size through the cavity.

The frequency range is from 0 to $300 \mathrm{~Hz}$. The sound level indicator is the mean quadratic pressure, expressed in $\mathrm{dB}$, and computed according to the following definition:

$$
L_{p}=10 \log _{10} \frac{\int_{\Omega_{\mathrm{F}}} p^{2} \mathrm{~d} V}{p_{0}^{2} \int_{\Omega_{\mathrm{F}}} \mathrm{d} V},
$$

where $p_{0}=20 \mu \mathrm{Pa}$ is the reference sound pressure in the air.

The frequency response functions in terms of the mean quadratic pressure in the cavity are plotted in Figure 6(a) for a few meshes above the six different non-conforming ones; they are compared with the reference solution. Because no damping is introduced for this problem, the plot is arbitrarily cut to $130 \mathrm{~dB}$ because the pressure level at resonances is not defined. For mesh 2, the frequency response function is also plotted when the tip enrichment is not use. The tip enrichment function for this mesh improves the accuracy of the solution. Mesh 3 gives a good accuracy compared with the reference solution.

A mean dB-difference (denoted by $\zeta$ ) is computed for each case using the following simple formulae

$$
\zeta=\frac{\sum \mid \text { dB-difference } \mid}{\text { Number of frequency steps }} .
$$


The mean $\mathrm{dB}$-difference between the reference frequency response function and the six different frequency response functions of the six non-conforming meshes is shown in Figure 6(b) versus the number of DOFS. The mean dB-difference is also computed when the tip enrichment is not use. For a coarse mesh (mesh 1), the mean dB-difference is quite high and the use of the tip enrichment does not improve the accuracy. For a fine mesh (meshes 5 and 6), the tip enrichment does not improve the accuracy because this enrichment is local to the structure tip. For intermediate size of mesh, the tip enrichment improves the accuracy of the solution.

The pressure field of the reference solution at $f=210 \mathrm{~Hz}$ is shown in Figure 7(a). Because there is no damping in this application, this frequency is arbitrarily chosen to be far enough from an eigenfrequency. The solution obtained by the proposed approach and for the fine mesh 6 is not plotted because it is visually the same. However, in order to compare the two solutions, the pressure at the structure tip is compared. It is plotted at the frequency of $210 \mathrm{~Hz}$ in Figure 7(b) for the different

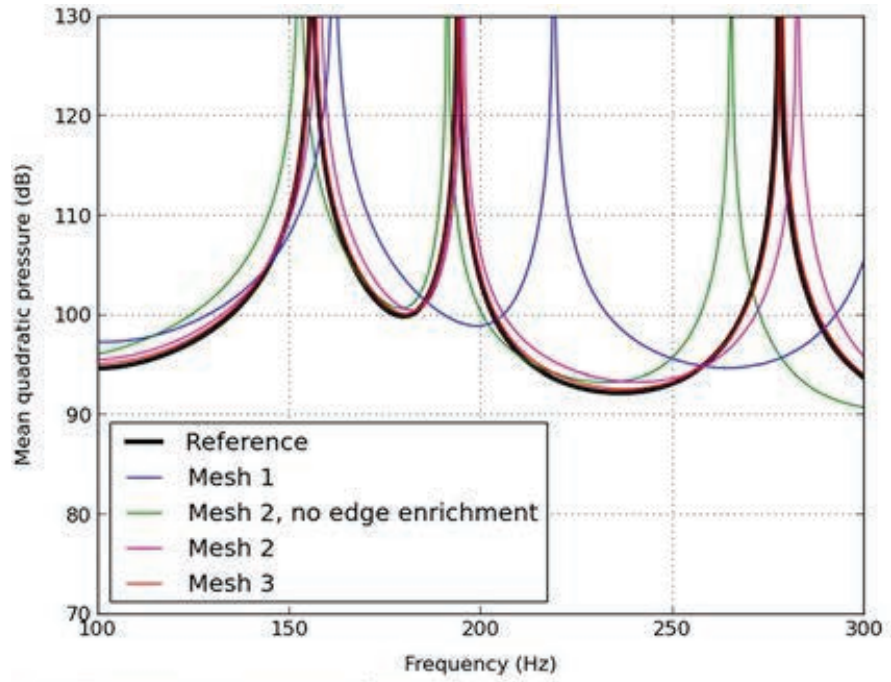

(a) Frequency response function

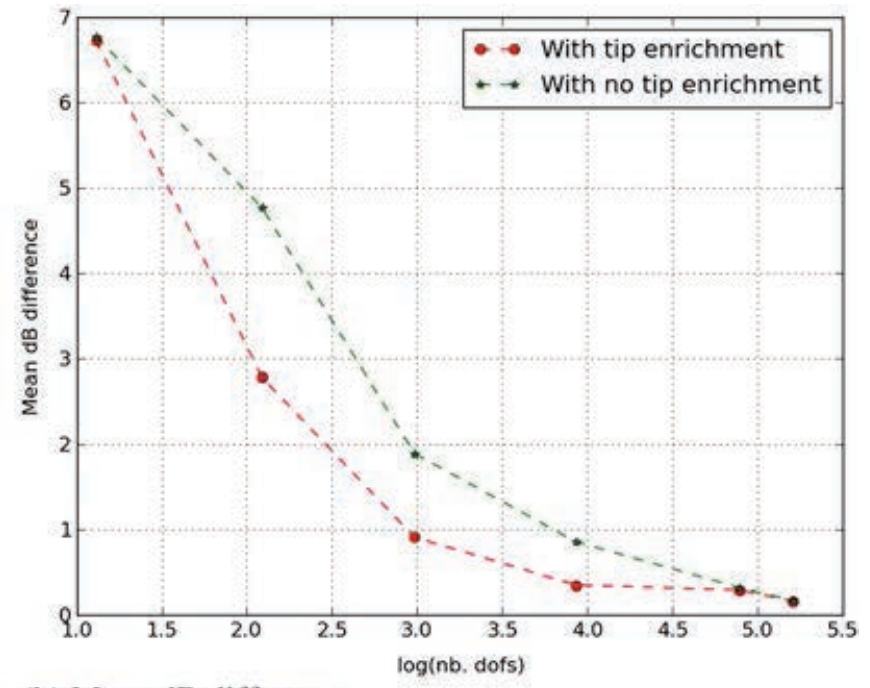

(b) Mean dB difference

Figure 6. Results for the separated cavity.
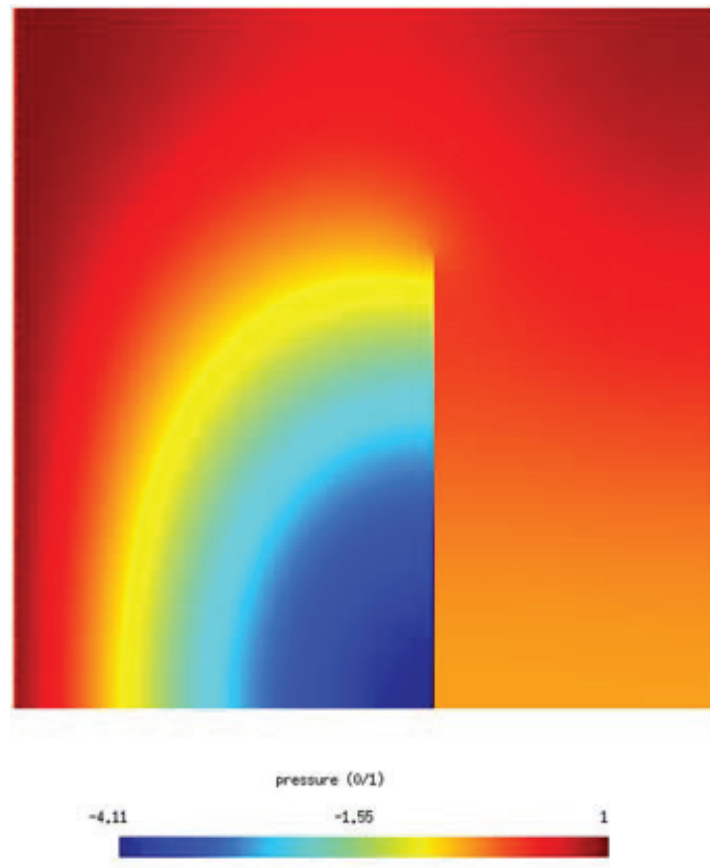

(a) Pressure field of the reference solution (Pa)

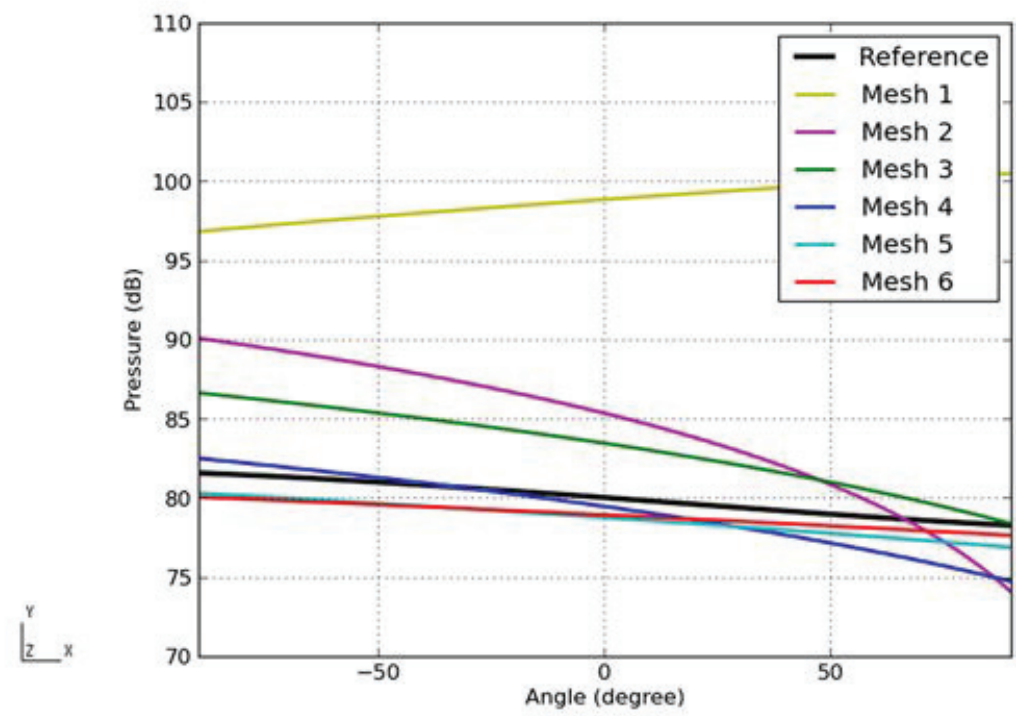

(b) Pressure at tip

Figure 7. Pressure field for the separated cavity at $f=210 \mathrm{~Hz}$. 

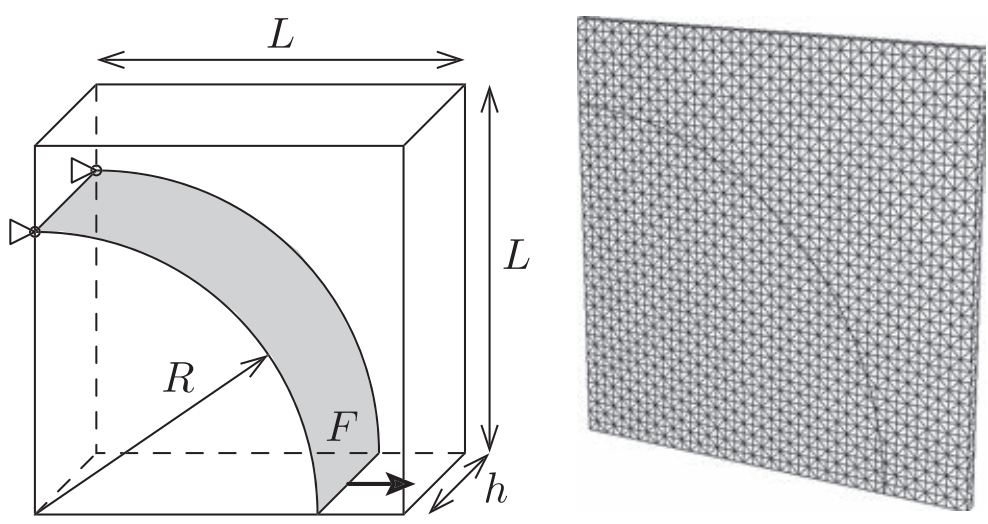

Figure 8. One-fourth of a circular structure ring in an acoustic cavity.

Table III. Data of the circular structure ring in an acoustic cavity.

\begin{tabular}{lclc}
\hline \multicolumn{1}{c}{ Fluid } & \multicolumn{2}{c}{ Structure } \\
\hline$L(\mathrm{~m})$ & 1 & $E(\mathrm{~Pa})$ & $70 \times 10^{9}$ \\
$h(\mathrm{~m})$ & 0.03 & $v$ & 0.27 \\
$c_{0}\left(\mathrm{~m} \mathrm{~s}^{-1}\right)$ & 340 & $\rho_{S}\left(\mathrm{~kg} \mathrm{~m}^{-3}\right)$ & 2700 \\
$\rho_{F}\left(\mathrm{~kg} \mathrm{~m}^{-3}\right)$ & 1.2 & $e$, thickness $(\mathrm{m})$ & $6 \times 10^{-3}$ \\
Fluid elements & 10086 & $R$, radius $(\mathrm{m})$ & 0.8 \\
Fluid nodes & 3528 & $F(\mathrm{~N})$ & 1 \\
Enriched elements & 336 & Structure elements & 650 \\
Enriched nodes & 228 & Structure nodes & 440 \\
\hline
\end{tabular}

meshes and compared with the one obtained along the semicircle for the reference solution. The pressure (in $\mathrm{dB}$ ) is plotted in terms of the angle $\theta$ defined in Figure 3 varying from $-90^{\circ}$ to $+90^{\circ}$. The convergence of the proposed method with a tip enrichment can be clearly seen. However, the solution does not converge (red curve, Mesh 6) to the reference one (black curve). This is probably because the structure has a non-zero thickness in the reference model, whereas it has a zero thickness in the non-conforming model.

This academic application shows that the tip enrichment improves the solution of the proposed method. This tip enrichment enables to extend the scope of the applications of this XFEM-based method for structural-acoustic coupled problems; compared with the first work proposed by the author [18], it is now possible to embed structures with free edges in the acoustic cavity.

\subsection{Convergence study of the reduced basis on a circular ring structure in an acoustic cavity}

One-fourth of a circular ring structure in a thin square acoustic cavity bounded by rigid walls is considered (Figure 8 and Table III). Boundary conditions as well as load are chosen such that the solution of the problem is two-dimensional. The structure is simply supported on its upper left boundary, the displacement along the cavity thickness direction is fixed for the whole structure. A harmonic load of amplitude $1 \mathrm{~N}$ is applied on the other side. Because the acoustic cavity is separated into two different cavities by the structure, no tip enrichment is used in this application. The chosen mesh is shown in [18] to be fine enough to give accurate results in the frequency range of interest (Table III and Figure 8). The studied frequency range is $1-600 \mathrm{~Hz}$, involving seven structure modes and 12 fluid modes of the empty cavity. The structural modal damping factor is chosen to be equal to 0.02 for all the modes. The first six structural modes are presented in Figure 9. The meshes and the post-processing are performed with Gmsh [38].

The convergence of the XFEM non-reduced method (system of Equation (6)) has been proven by comparisons with a standard compatible mesh approach in [18]. The reference solution in the following is then based on the XFEM non-reduced approach to only get the error coming from the reduction and not the one coming from the discretization. This reference solution is thus obtained by solving the system of Equation (15) using the same mesh with 100 structure modes enriched by 


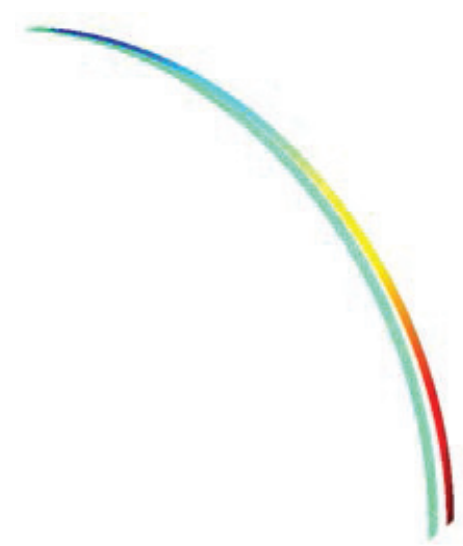

(a) mode $1,10.7 \mathrm{~Hz}$

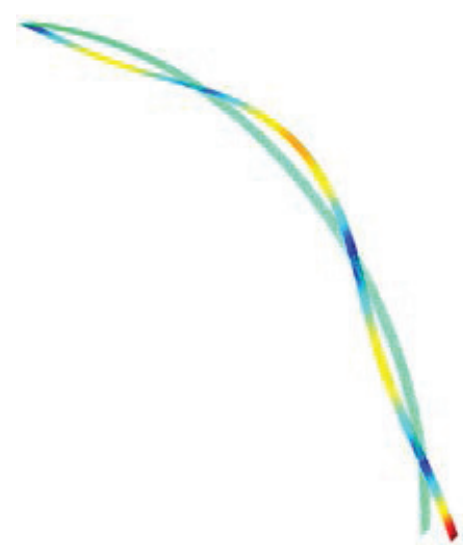

(d) mode $4,154.3 \mathrm{~Hz}$

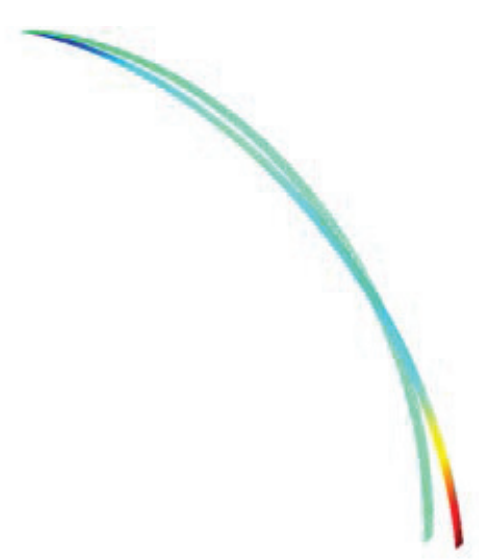

(b) mode $2,40.4 \mathrm{~Hz}$

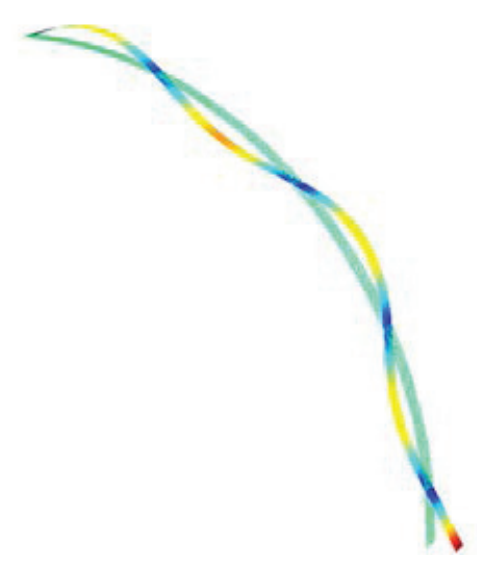

(e) mode $5,237.8 \mathrm{~Hz}$

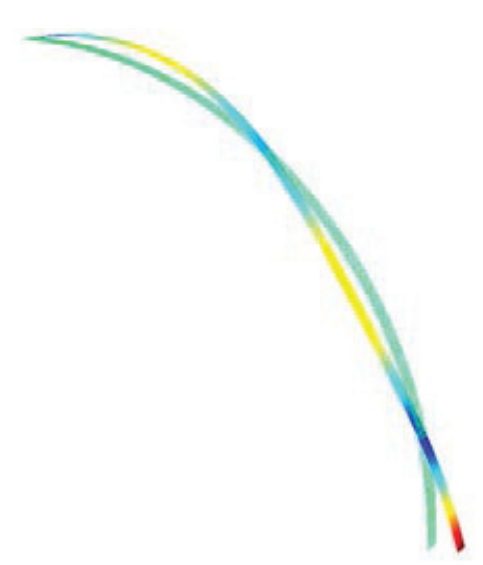

(c) mode $3,88.5 \mathrm{~Hz}$

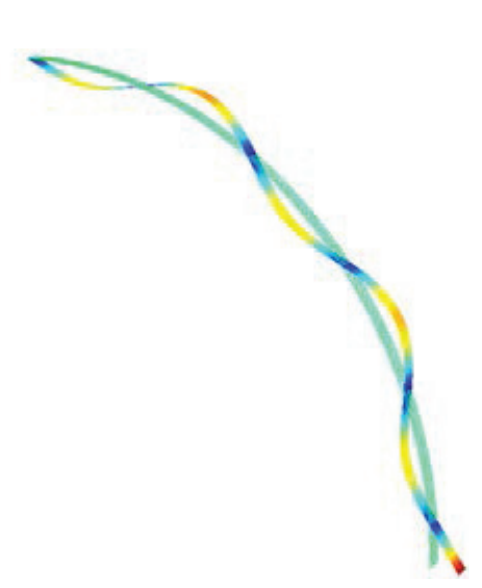

(f) mode $6,339.1 \mathrm{~Hz}$

Figure 9. Structure modal basis of the circular ring.

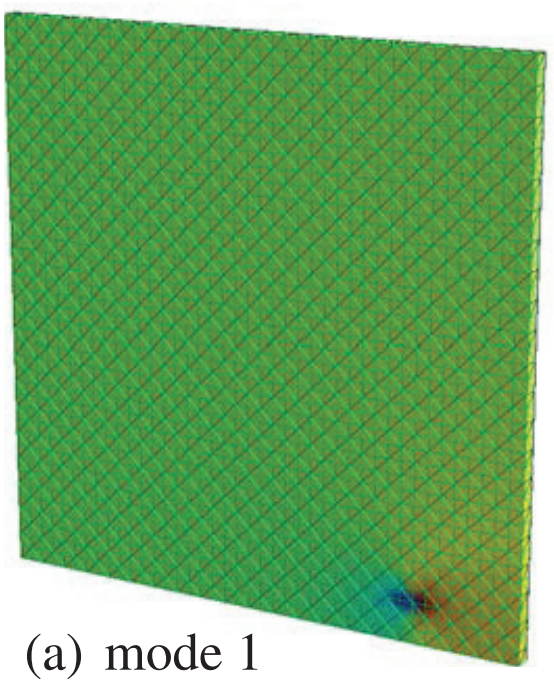

(a) mode 1

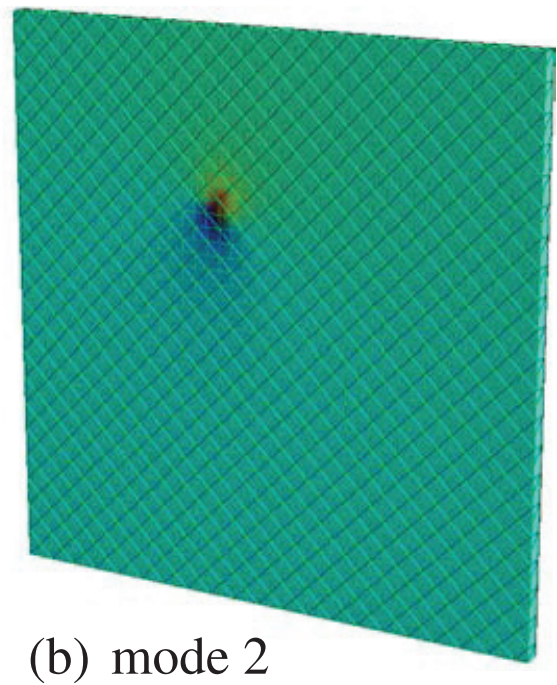

(b) mode 2

Figure 10. Example of two static modes for the circular ring in an acoustic cavity among 228 static modes.

the static solution. The eigenfrequency of the $100^{\text {th }}$ mode is around $25,100 \mathrm{~Hz}$, which is far more than $600 \mathrm{~Hz}$, meaning that taking 100 structure modes gives a converged solution.

Two static modes $\Psi_{\mathrm{F}}^{i}$ are plotted in Figure 10. These vectors can be seen as a global XFEM enrichment of the fluid basis in order to take into account the structure in the coupled problem, there are 228 static modes in this application (228 enriched nodes).

6.2.1. Convergence of the fluid basis. In order to study the convergence of the reduction method in terms of fluid reduced basis, the number of structure modes is kept as a constant, 100 modes are chosen. Five cases are studied, containing 5, 10, 20, 40, and 80 fluid modes in the basis $\boldsymbol{\Phi}_{\mathrm{Fp}}$, they are named, respectively, in the following as $5 \mathrm{~F}, 10 \mathrm{~F}, 20 \mathrm{~F}, 40 \mathrm{~F}$, and $80 \mathrm{~F}$. 
The first eight modes of the fluid basis $\boldsymbol{\Phi}_{\mathrm{Fp}}$ of the cavity with no structure inside are presented in Figure 11.

The frequency response functions are compared in Figure 12(a) with the reference solution for cases $5 \mathrm{~F}, 20 \mathrm{~F}$, and $80 \mathrm{~F}$. In order to quantify the influence of the number of fluid modes, an absolute value of the dB-difference with the reference solution is computed for each case and for each frequency. This means that $\mathrm{dB}$-difference is compared with the ratio between the highest eigenfrequency in the fluid basis (depending on the number of fluid modes) over the highest observed frequency $(600 \mathrm{~Hz}$ in this case) in Figure 12(b). For this application, choosing the fluid modes with an eigenfrequency up to twice the observed frequency gives accurate results with a mean dB-difference less than $1 \mathrm{~dB}$ (case 40F).

6.2.2. Convergence of the structure basis. In order to study the convergence of the reduction method in terms of structure reduced basis, the fluid is not reduced and the system of Equation (15) is solved. Four cases are studied, containing 2, 4, 8, and 16 structure modes in the basis $\boldsymbol{\Phi}_{\mathrm{Sm}}$, they

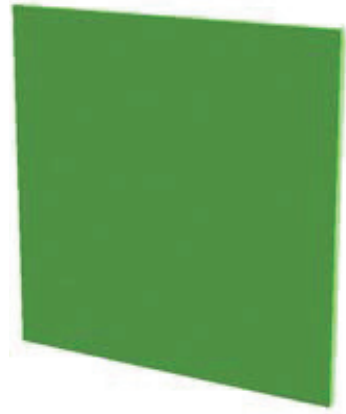

(a) mode $1,0.0 \mathrm{~Hz}$

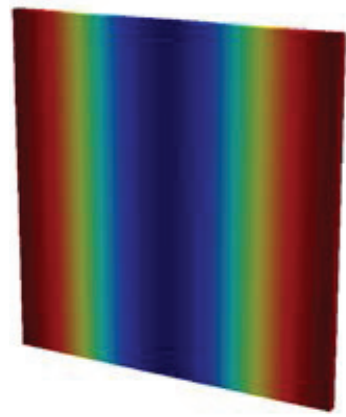

(e) mode $5,338.2 \mathrm{~Hz}$

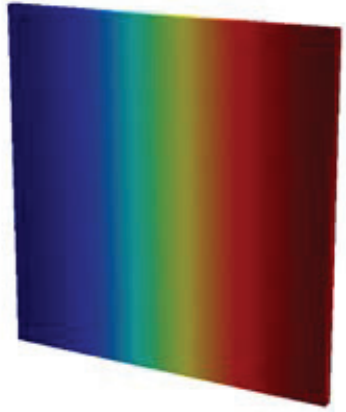

(b) mode 2, $168.9 \mathrm{~Hz}$

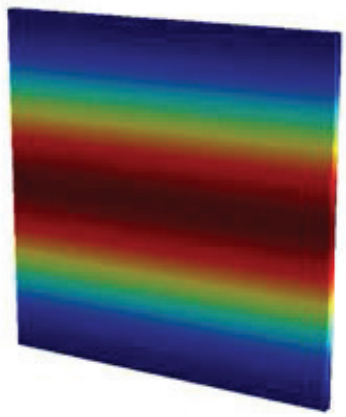

(f) mode $6,340.3 \mathrm{~Hz}$

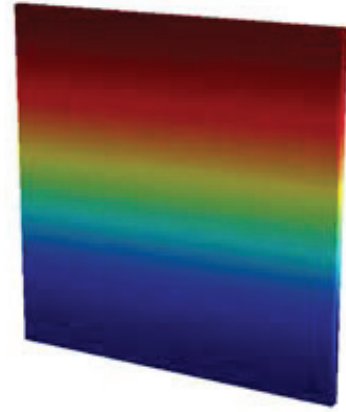

(c) mode $3,170.0 \mathrm{~Hz}$

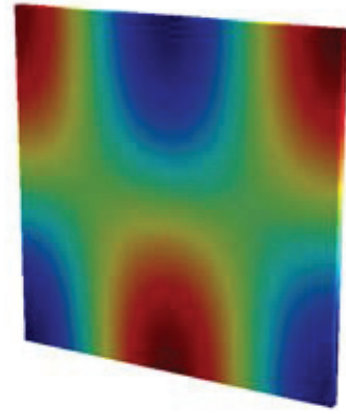

(g) mode 7, $378.6 \mathrm{~Hz}$

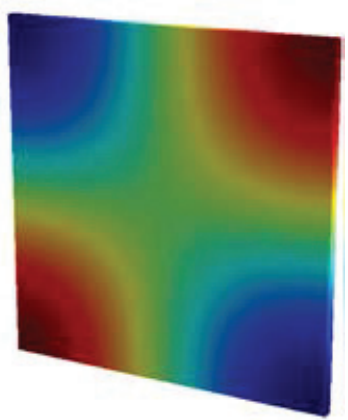

(d) mode $4,239.7 \mathrm{~Hz}$

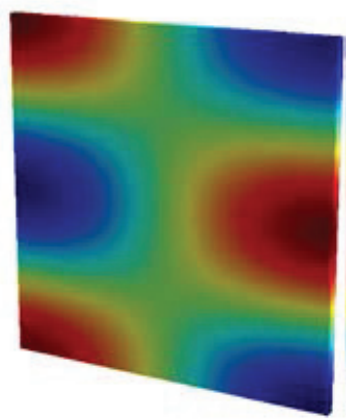

(h) mode $8,380.1 \mathrm{~Hz}$

Figure 11. Fluid modal basis of the empty cavity for the circular ring problem.

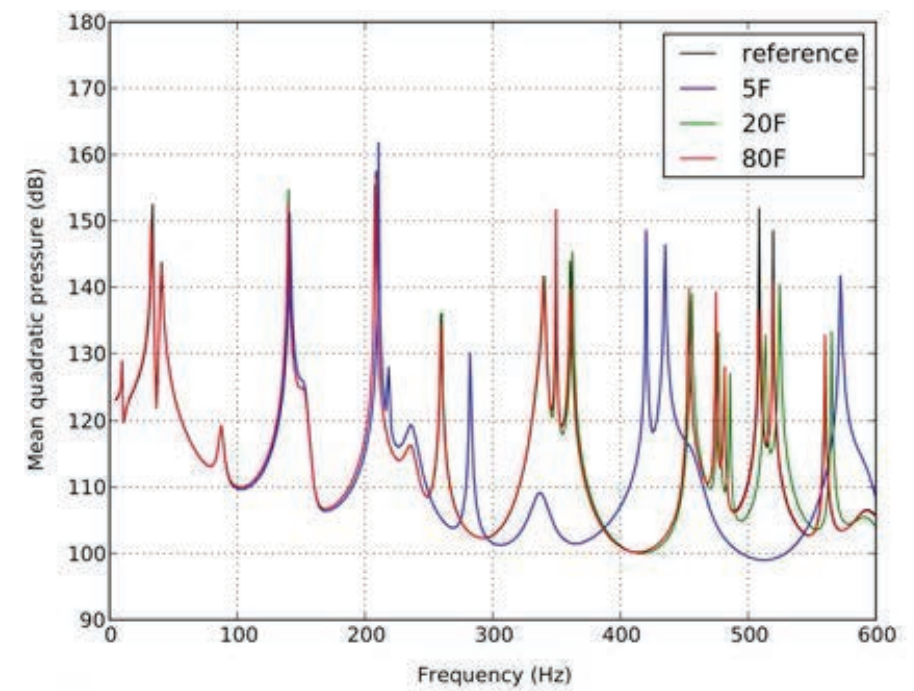

(a) Frequency response function

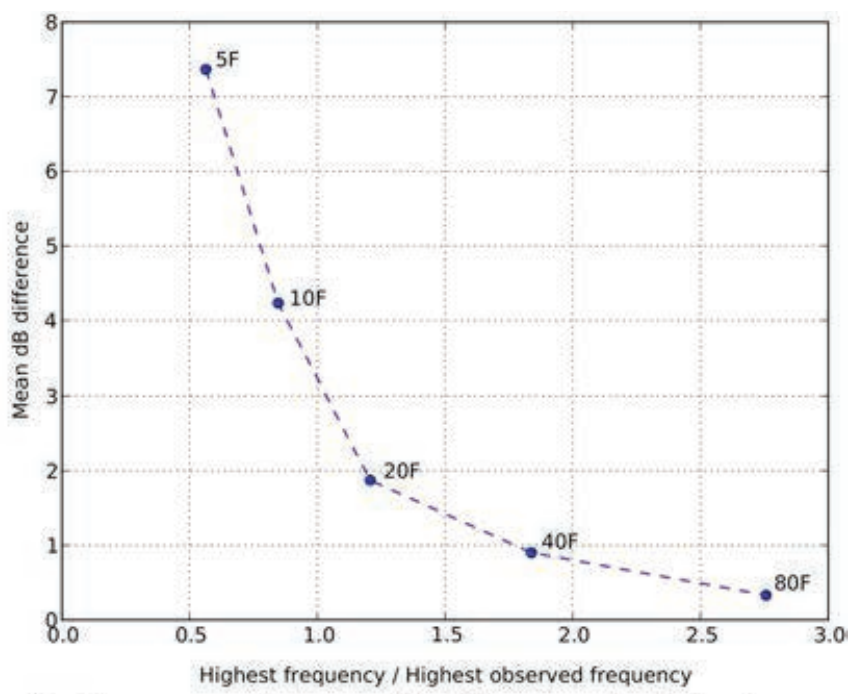

(b) Convergence in term of last frequency in the basis

Figure 12. Fluid basis convergence for the circular ring problem. 


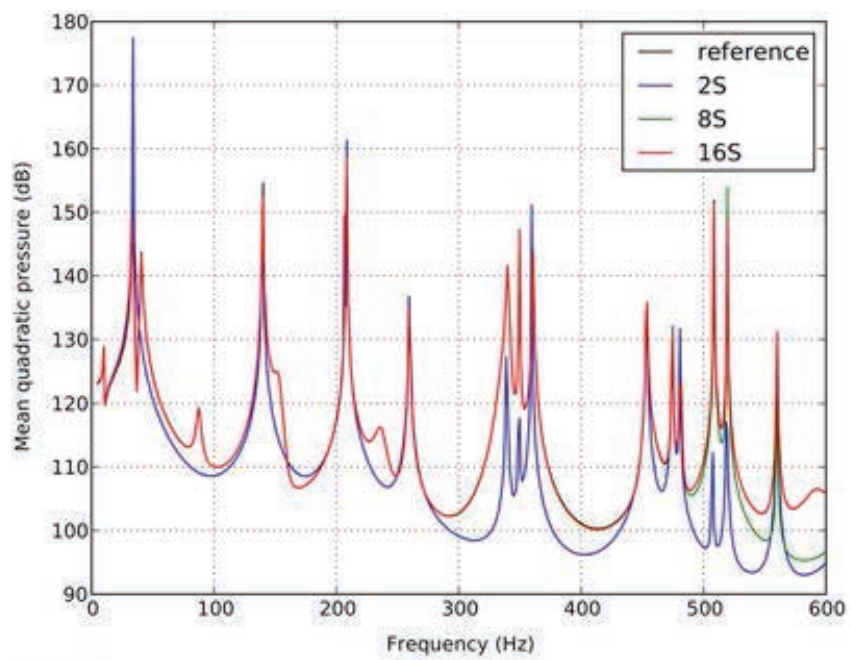

(a) Frequency response function

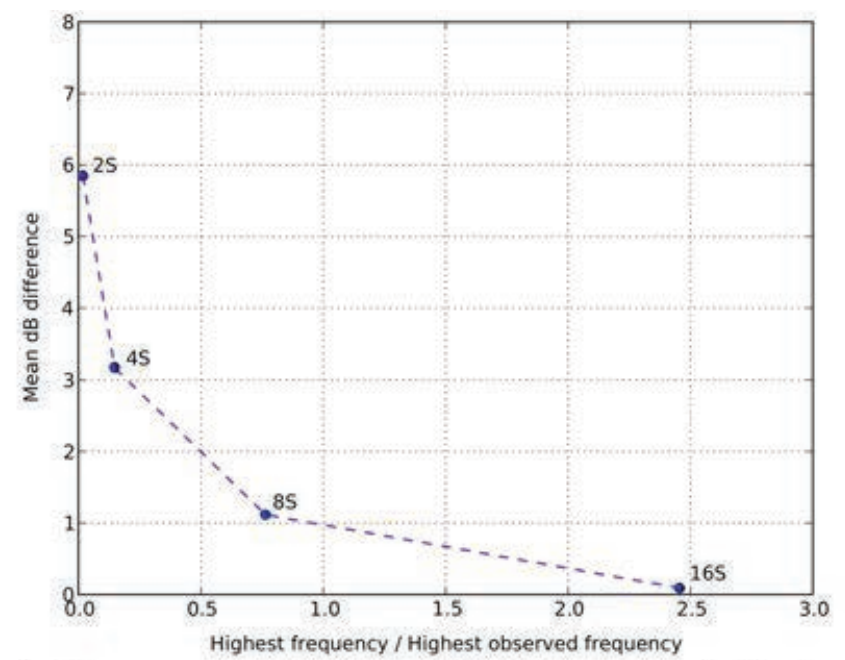

(a) Convergence in term of last frequency in the basis

Figure 13. Structure basis convergence for the circular ring problem, different structure bases.

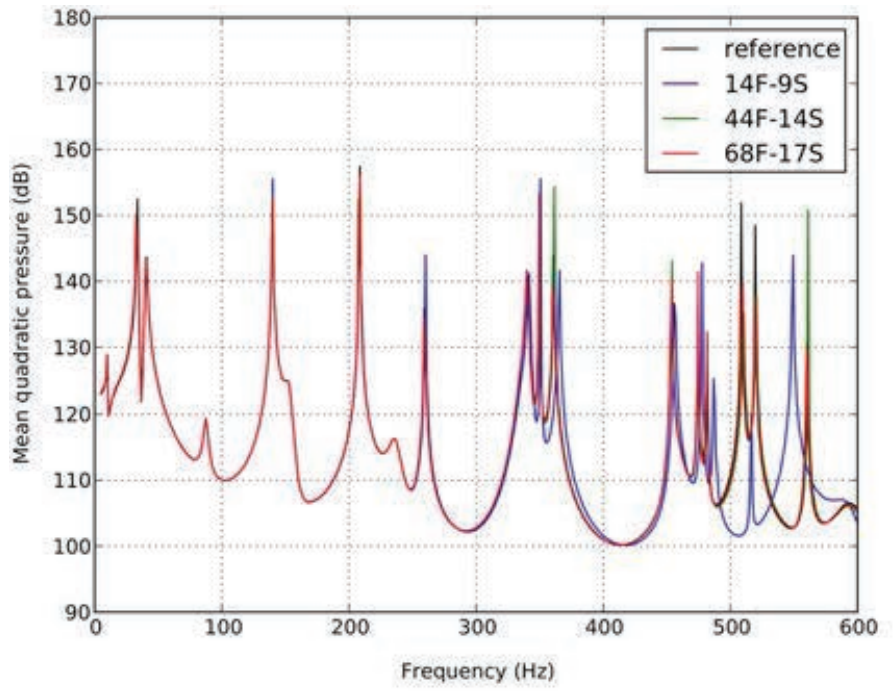

(a) Frequency response function

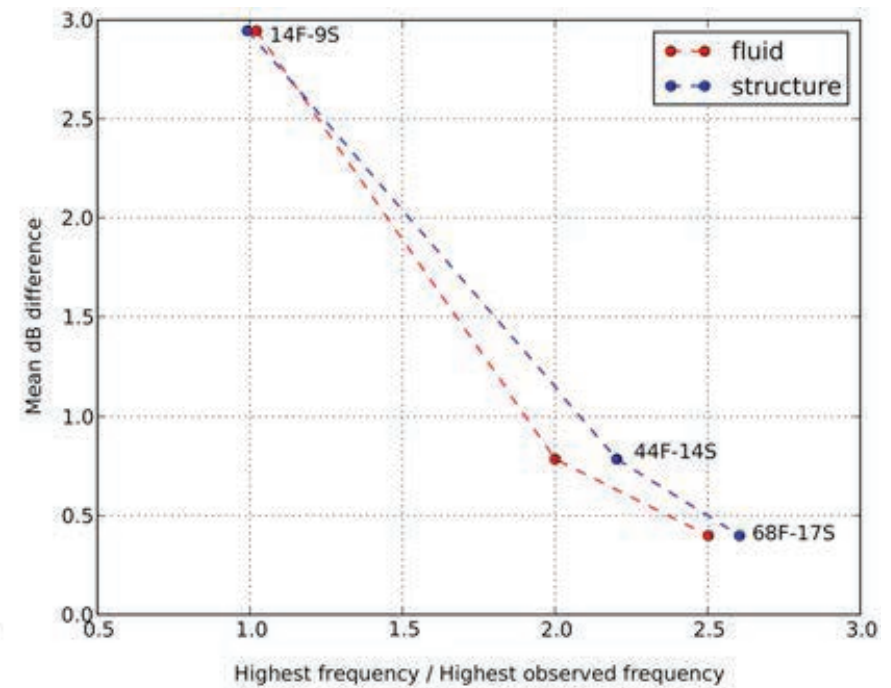

(a) Convergence in term of last frequency in the basis

Figure 14. Convergence for the circular ring problem.

are named, respectively, in the following as $2 \mathrm{~S}, 4 \mathrm{~S}, 8 \mathrm{~S}$, and $16 \mathrm{~S}$. The static solution is added to each case in order to form the reduced basis $\boldsymbol{\Phi}_{\mathrm{Sn}}$.

The frequency response functions for the cases $2 \mathrm{~S}, 8 \mathrm{~S}$, and $16 \mathrm{~S}$ are plotted in Figure 13(a) and compared with the reference solution. The convergence can be clearly seen because only the two first resonances are correctly represented with the case $2 \mathrm{~S}$, whereas the case $8 \mathrm{~S}$ gives accurate results up to about $500 \mathrm{~Hz}$. The mean dB-difference is compared with the ratio between the highest eigenfrequency in the structure basis (depending on the number of structure modes) over the highest observed frequency $(600 \mathrm{~Hz}$ in this case) in Figure 13(b). For the case 16S, which gives very good results, the highest eigenfrequency of the structural basis is more than twice larger than the observed frequency.

6.2.3. Convergence of both fluid and structure basis. In order to study the convergence of the solution to both the fluid and the structure basis, three cases are studied: 14F-9S, 44F-14S, and 68F-17S. The frequency response functions are compared with the reference in Figure 14(a). The fluid pressure field and the structural displacement are presented in Figure 15(a) at $336 \mathrm{~Hz}$ for the case 44F-14S. The structural displacement is plotted with an amplification factor of 1000. The XFEM results show a discontinuous jump at the interface because the plot routine does not take into account the Heaviside enrichment in the enriched elements, whereas the enrichment is used to compute the real pressure at an enriched node according to Equation (2).

The mean $\mathrm{dB}$-difference is compared with the ratio between the highest eigenfrequency of the fluid basis and of the structure basis over the highest observed frequency (600 Hz in this case) in 


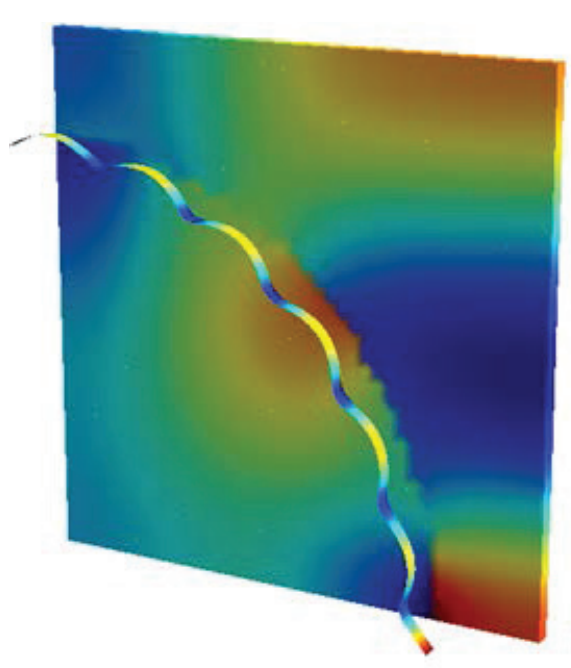

(a) Fluid pressure and structure displacement at $336 \mathrm{~Hz}$

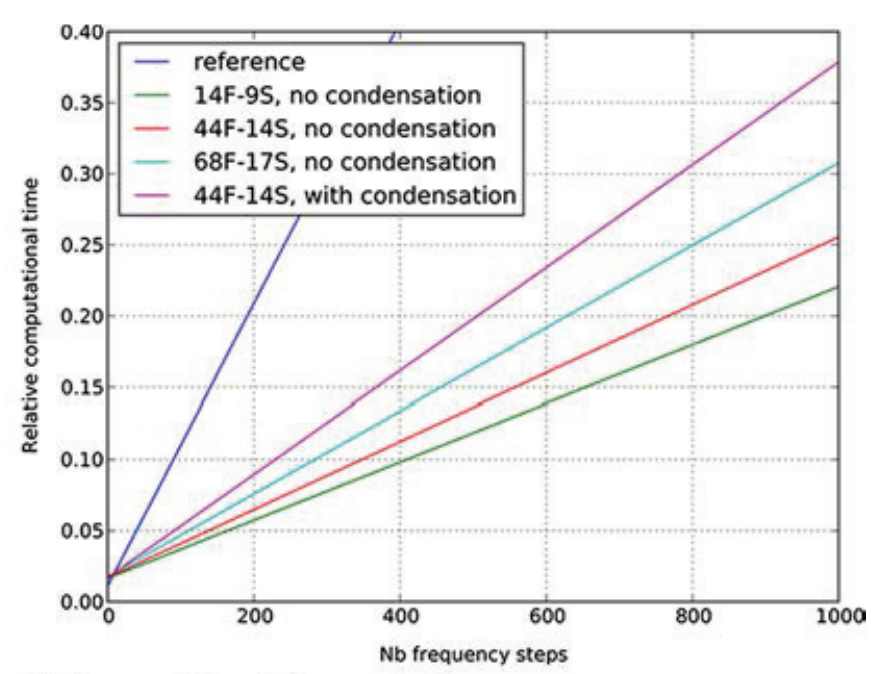

(b) Computational time reduction

Figure 15. Results for the circular ring problem.

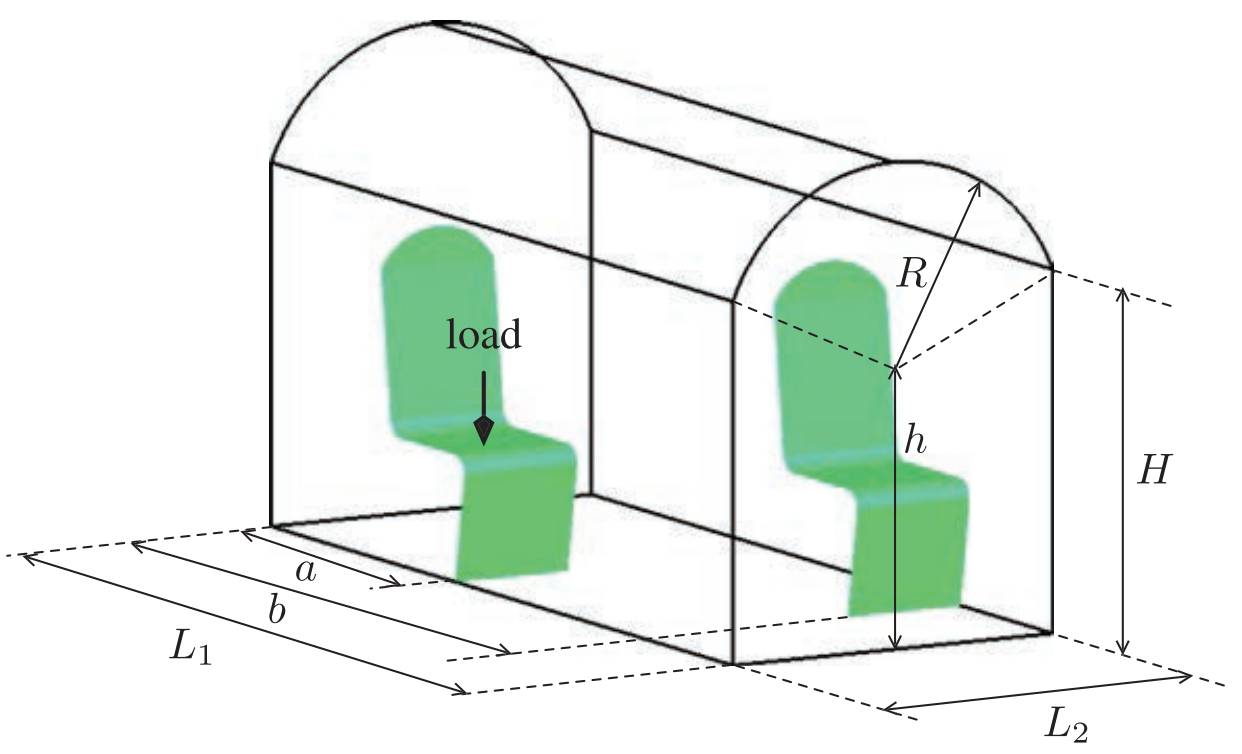

Figure 16. Two structures in an acoustic cavity.

Figure 14(b). These three cases are in good agreement with the previous conclusion concerning the choice of the number of modes in the reduced bases, namely, twice the observed frequency for the reduced basis gives accurate results (case 44F-14S).

The computational time versus the number of frequency steps is presented in Figure 15(b), it is normalized to one of the reference solutions. A fixed time is needed at the beginning in order to form the reduced system. The computational time for the optimal case $44 \mathrm{~F}-14 \mathrm{~S}$ represents only $25 \%$ of the reference time for 1000 computed frequency steps when using the non-condensed version (gain factor of 4 ), whereas it is about $40 \%$ with the condensed version. The computational time is not really affected by the increasing number of modes.

\subsection{Study of the influence of the positions of two structures in an acoustic cavity}

6.3.1. Definition of the geometry and the meshes. The acoustic cavity is described in Figure 16, and the numerical values are given in Table IV. Two identical structures are immersed in this acoustic cavity. Their geometries are defined in Figure 17 in a local coordinate system. The two structures are arbitrarily localized in the cavity according to the $a$ and $b$ parameters, which give the positions of point number 1 for each structure in the cavity. A harmonic load is applied in $z$-direction on point number 10 of the structure localized by the parameter $a$. The fluid mesh $(22,473$ nodes; 125,327 tetrahedral elements) is shown in Figure 18(a), whereas the structure mesh (1894 nodes; 3613 DKT elements) is plotted in Figure 18(b). These two meshes have been chosen after a convergence study, 
Table IV. Data of the acoustic cavity with two structures.

\begin{tabular}{lccc}
\hline & \multicolumn{2}{c}{ Fluid } & \multicolumn{2}{c}{ Structure } \\
\hline$L_{1}(\mathrm{~m})$ & 5 & $v$ & $75,000 \times 10^{6}$ \\
$L_{2}(\mathrm{~m})$ & 2 & $\rho_{S}\left(\mathrm{~kg} \mathrm{~m}^{-3}\right)$ & 0.33 \\
$H(\mathrm{~m})$ & 2 & $e$, thickness $(\mathrm{m})$ & $15 \times 10^{-3}$ \\
$h(\mathrm{~m})$ & 1.6 & $l(\mathrm{~m})$ & 0.7 \\
$R$ & $\sqrt{(H-h)^{2}+L_{2}^{2} / 4}$ & & \\
$c_{0}\left(\mathrm{~m} \mathrm{~s}^{-1}\right)$ & 340 & & \\
$\rho_{F}\left(\mathrm{~kg} \mathrm{~m}^{-3}\right)$ & 1.2 & & \\
$a, b(\mathrm{~m})$ & Variable & \\
\hline
\end{tabular}
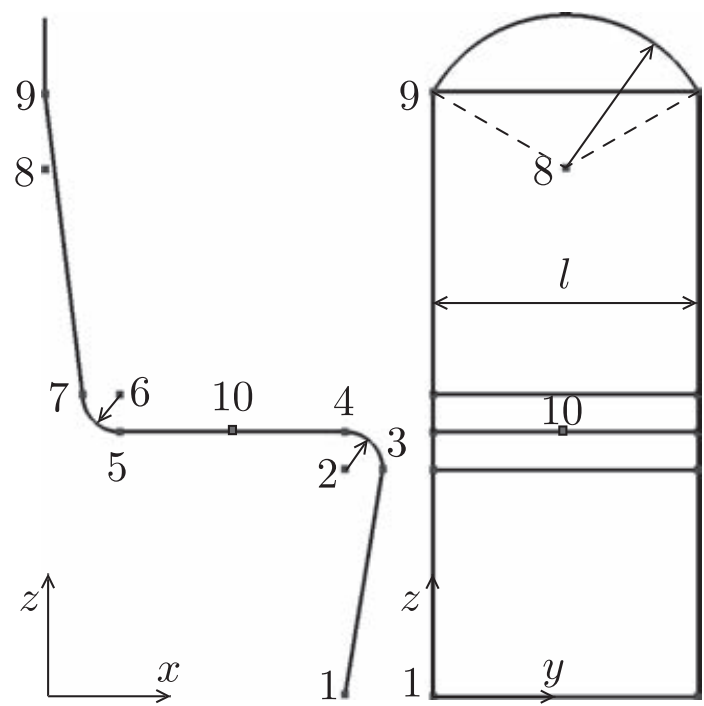

\begin{tabular}{llll}
\hline Point & $\mathrm{x}(\mathrm{m})$ & $\mathrm{y}(\mathrm{m})$ & $\mathrm{z}(\mathrm{m})$ \\
\hline 1 & 0.8 & 0.0 & 0.0 \\
2 & 0.8 & 0.0 & 0.6 \\
3 & 0.9 & 0.0 & 0.6 \\
4 & 0.8 & 0.0 & 0.7 \\
5 & 0.2 & 0.0 & 0.7 \\
6 & 0.2 & 0.0 & 0.8 \\
7 & 0.1 & 0.0 & 0.8 \\
8 & 0.0 & 0.35 & 1.4 \\
9 & 0.0 & 0.0 & 1.6 \\
10 & 0.5 & 0.35 & 0.7 \\
\hline
\end{tabular}

Figure 17. Description of one structure.

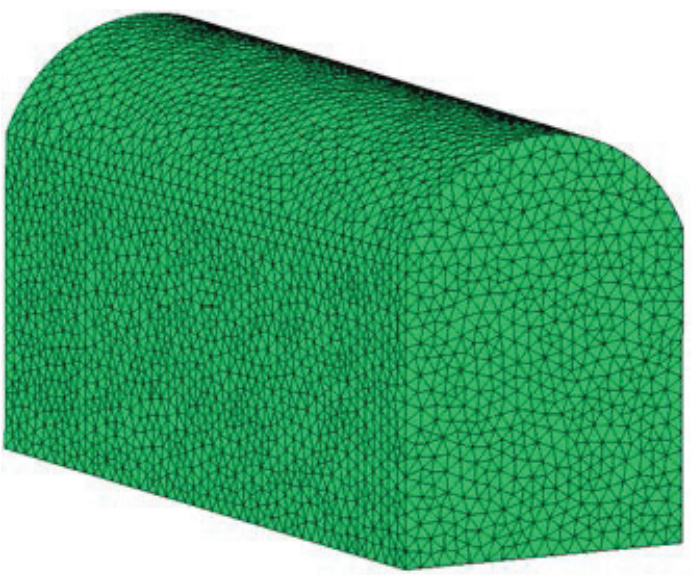

(a) Fluid

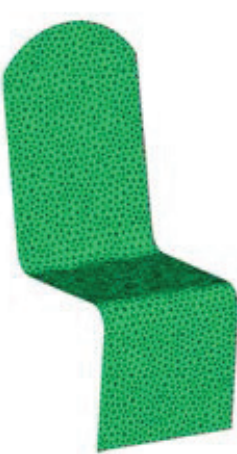

(b) Structure

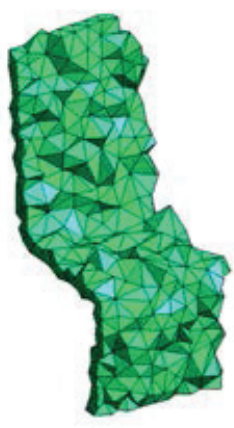

(c) Enriched mesh

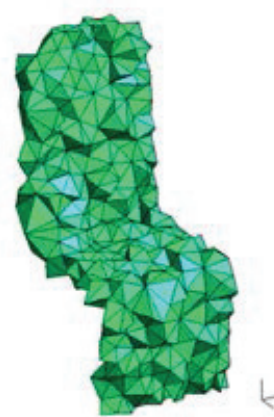

Figure 18. Details of the meshes: fluid mesh $(22,473$ nodes/125,327 tetrahedral elements); structure mesh (1894 nodes/3613 DKT elements); enriched mesh (771 nodes/1917 tetrahedral elements).

they are fine enough to compute correctly the solution in the studied frequency range. A modal damping factor of 0.02 is introduced to all structure modes. The pressure field is shown for two frequencies in Figure 19.

6.3.2. Convergence study. The convergence study in terms of the number of modes taken into account into the reduced basis is realized for one specific configuration: $a=2 \mathrm{~m}$ and $b=4 \mathrm{~m}$. The enriched elements (771 nodes; 1917 elements), used as the interface between the fluid and the structure, are presented in Figure 18(c). The number of enriched nodes is 771, meaning that the size 


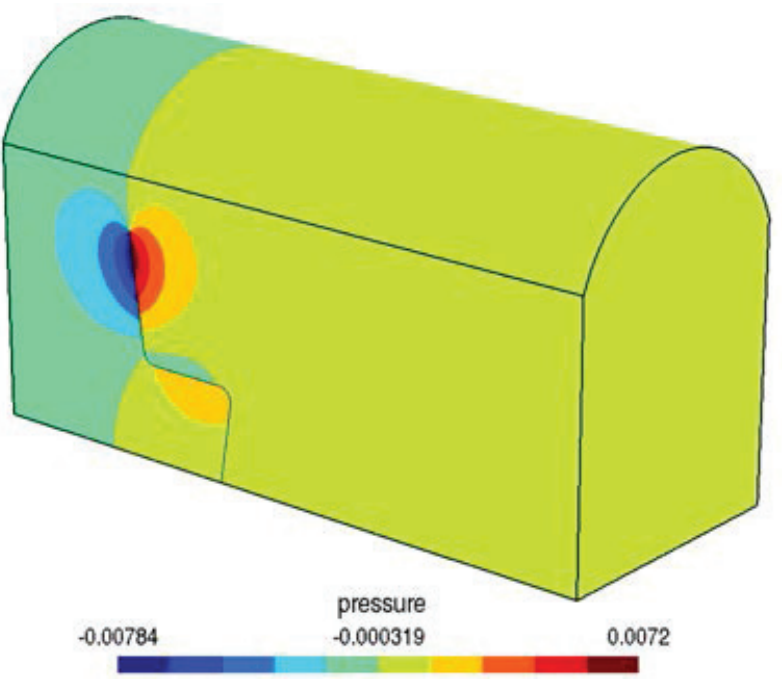

(a) Pressure field at $27.1 \mathrm{~Hz}$

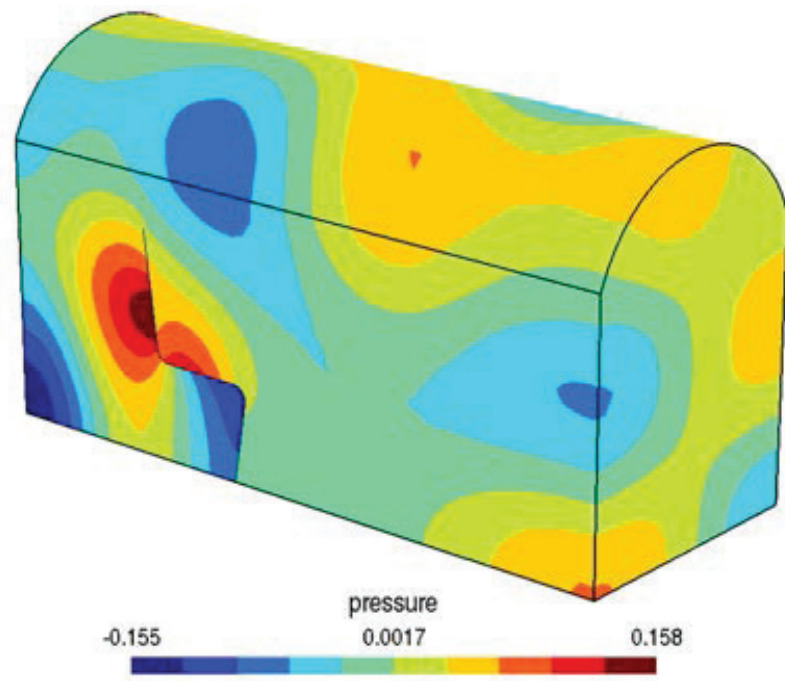

(b) Pressure field at $168.6 \mathrm{~Hz}$

Figure 19. Pressure field in the acoustic cavity for two specific frequencies.

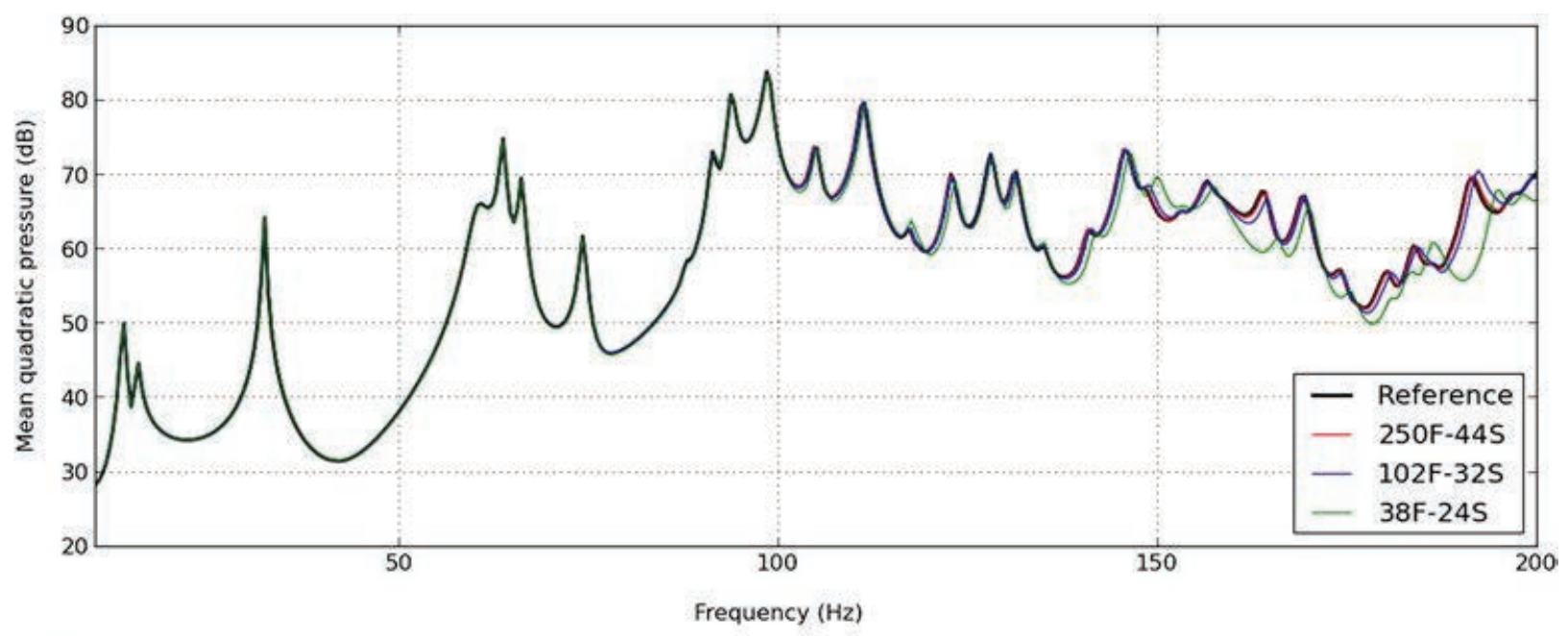

(a) Frequency response function

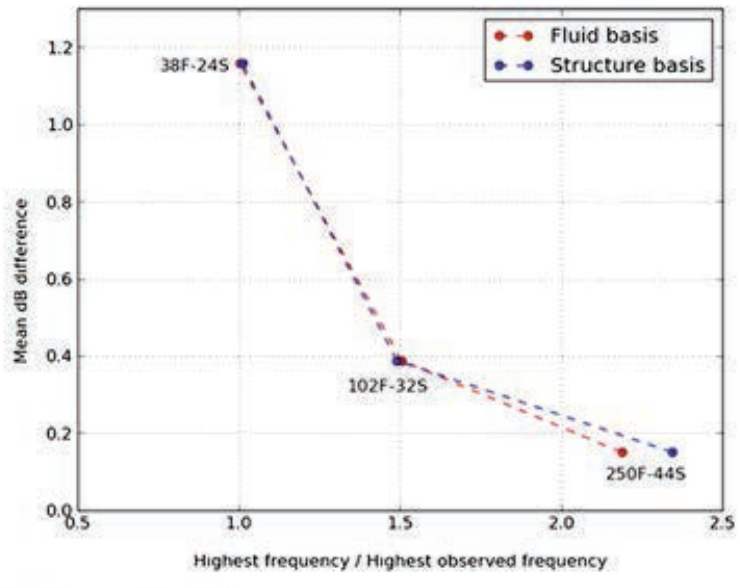

(b) Mean dB-difference

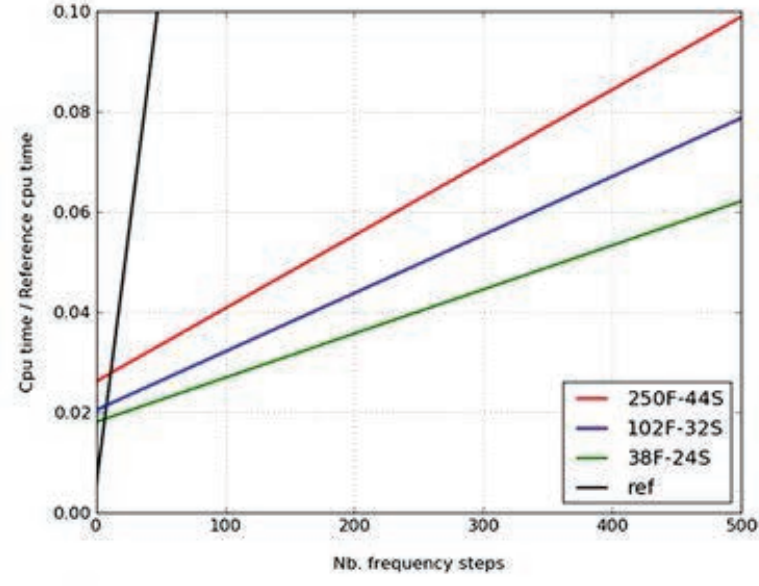

(c) Computational time comparison

Figure 20. Results.

of the reduced problem is of this order. This represents a significant reduction compared with the number of nodes of the empty cavity mesh (22,473 nodes).

Three reduced basis are used in order to show the convergence of the method, involving 38 fluid modes and 24 structure modes, 102 fluid modes and 32 structure modes, and finally 250 fluid modes and 44 structure modes. These three cases are denoted, respectively, 38F-24S, 102F-32S, and 250F- 


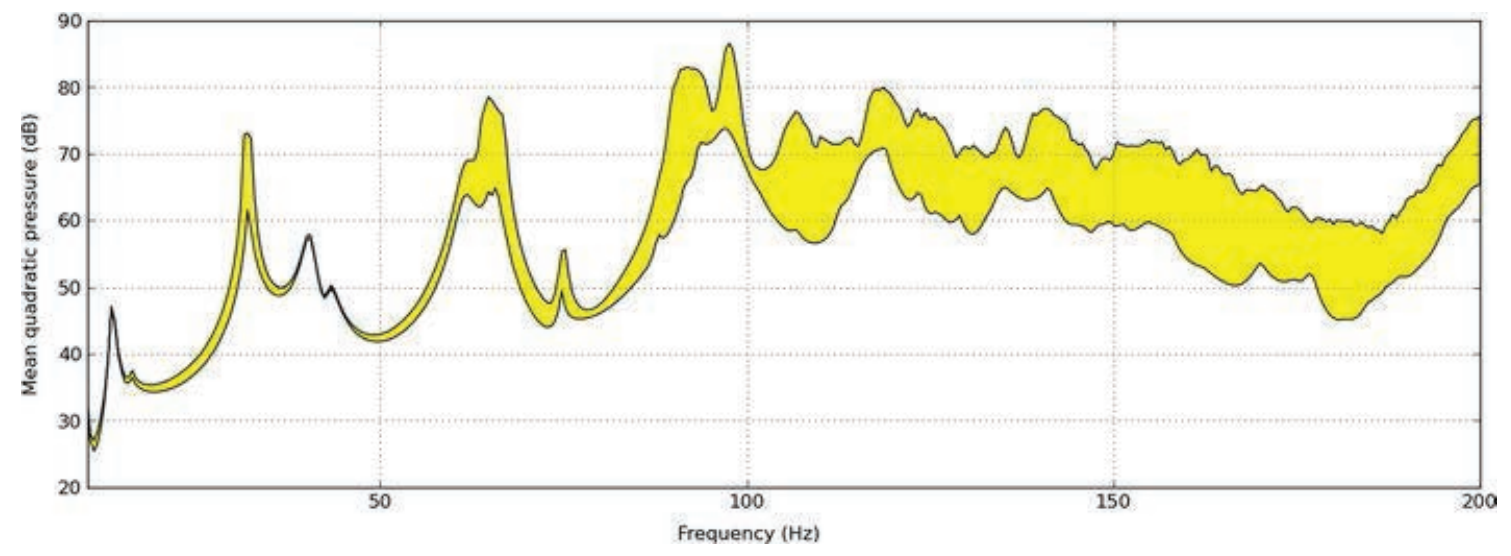

Figure 21. Frequency response function envelope of the parametric study.

44S. The eigenfrequency of the last mode on the basis corresponds approximately, for each case, to $1,1.5$, and 2 times the observed frequency ( $200 \mathrm{~Hz}$ in this application).

The frequency response functions in terms of the mean quadratic pressure expressed in $\mathrm{dB}$ in the acoustic cavity for the three basis are plotted in Figure 20(a). They are compared with a reference solution obtained with no reduction in the fluid, whereas 44 structure modes are taken into account in the structure basis. The differences are more important for large frequencies: basis $38 \mathrm{~F}-24 \mathrm{~S}$ shows differences from 100 to $200 \mathrm{~Hz}$, basis 102F-32S gives good results up to $150 \mathrm{~Hz}$, whereas basis $250 \mathrm{~F}-44 \mathrm{~S}$ is accurate up to the highest observed frequency $(200 \mathrm{~Hz})$. The mean dB-difference is shown in Figure 20(b) for the three basis, which confirm the fact that the more modes are taken into account in the basis, the more accurate are the results. The computational time is plotted in Figure 20(c), this time is normalized to the reference time to compute 500 frequency steps. The computational time is divided by a factor of 10 for basis $250 \mathrm{~F}-44 \mathrm{~S}$, which gives very accurate results in the frequency range of interest.

6.3.3. Variation of the parameters. In order to show the ability of the method to perform a parametric study of the structure positions, the two structures are placed arbitrarily in the fluid cavity. Parameter $a$ varies from 2 to 3, whereas parameter $b$ varies from 3 to 4 . Eleven values of each parameter are chosen $(a \in[2.0,2.1, \ldots, 3.0]$ and $b \in[3.0,3.1, \ldots, 4.0])$, which leads to a total of 121 studied configurations. The fluid mesh as well as the structure mesh are the same as in the previous section. The reduced basis $250 \mathrm{~F}-44 \mathrm{~S}$ is chosen for all the computations, it is computed only once. A parallel computing strategy is used: eight processors computes eight different configurations at the same time, leading to an additional computational time saving. The envelope curves of the 121 frequency response functions are presented in Figure 21.

\section{CONCLUSIONS}

A reduced method based on XFEM is presented in this work in order to efficiently compute a structural-acoustic problem involving variations of the structures' positions and/or geometries. The XFEM enables to immerse the thin structures at arbitrary positions in the acoustic fluid without remeshing the whole domain. A structure tip enrichment is introduced in order to take into account the pressure variation at one geometric point. This effect cannot be modeled by a standard finite element approach if the structure is a shell.

The reduced proposed approach is based on a modal synthesis method. The enriched fluid nodes give a natural interface between the fluid and the structures. The internal fluid domain corresponds to the empty cavity with no structures. The advantage is that the modal basis of the fixed interface problem is the modal basis of the acoustic cavity with no structure, which can be thus computed independently of the immersed structures. The structural DOFS are projected on the structure modal basis decoupled from the fluid. A structural modal damping is added in this work. The size of the reduced system is the number of enriched fluid nodes increased by the number of fluid and structure modes taken into account. 
The different test cases show that the optimal choice of fluid and structure bases corresponds to the last eigenfrequencies of both bases taken as, at least, twice the observed frequency. The computational time is reduced by a factor from 2 to 10 depending on the problem. Indeed, the reduced system is full in part corresponding to the enriched DOFS (the larger part of it), whereas the not-reduced initial large system is sparse. Therefore, the computational time is not proportional to the size of the system. The numerical tests show that for large applications, it is better to not condense the internal fluid modes on the interface in order to save computational time. However, the computational time comparisons done in this work do not take into account the needed remeshing time of a standard compatible mesh approach: the real gain factor is larger.

Finally, a parametric study of the positions of two structures in an acoustic cavity demonstrates the full potential of the proposed reduced method.

\section{REFERENCES}

1. Boeing uses LMS SYSNOISE to predict acoustics of aircraft cabins. LMS news. February 19-21, 2003.

2. Cherng JG, Gang Y, Bonhard RB, French M. Characterization and validation of acoustic cavities of automotive vehicles. IMAC-XX, Conference on Structural Dynamics, Los Angeles, CA, 2002; 290-294.

3. Allard J-F. Propagation of Sound in Porous Media: Modelling Sound Absorbing Materials. Elsevier Applied Science: London, New York, 1993.

4. Rao MD. Recent applications of viscoelastic damping for noise control in automobiles and commercial airplanes. Journal of Sound and Vibration 2003; 262(3):457-474.

5. Ohayon R. Reduced models for fluid-structure interaction problems. International Journal for Numerical Methods in Engineering 2004; 60(1):139-152.

6. Bobillot $\mathrm{A}$, Balmès E. Iterative techniques for eigenvalue solutions of damped structures coupled with fluids. AIAA Journal 2002-1391, 43rd AIAA/ASME/ASCE/AHS/ASC Structures, Structural Dynamics, and Materials Conference, Denver, Colorado, 2002.

7. Rouleau L, Deü J-F, Legay A, Sigrist J-F. Vibro-acoustic study of a viscoelastic sandwich ring immersed in water. Journal of Sound and Vibration 2012; 331(3):522-539.

8. Rumpler R, Legay A, Deü J-F. Performance of a restrained-interface substructuring FE model for reduction of structural-acoustic problems with poroelastic damping. Computers and structures 2011; 89(23-24):2233-2248.

9. Davidsson P, Sandberg G. A reduction method for structure-acoustic and poroelastic-acoustic problems using interface-dependent Lanczos vectors. Computer Methods in Applied Mechanics and Engineering 2006; 195(17-18):1933-1945.

10. Junge M, Brunner D, Becker J, Gaul L. Interface-reduction for the Craig-Bampton and Rubin method applied to FE-BE coupling with a large fluid-structure interface. International Journal for Numerical Methods in Engineering 2009; 77(12):1731-1752.

11. Tran QH, Ouisse M, Bouhaddi N. A robust component mode synthesis method for stochastic damped vibroacoustics. Mechanical Systems and Signal Processing 2010; 24(1):164-181.

12. Deü J-F, Larbi W, Ohayon R. Vibration and transient response of structural acoustic interior coupled systems with dissipative interface. Computer Methods in Applied Mechanics and Engineering 2008; 197(51-52):4894-4905.

13. Bermúdez A, Rodríguez R. Modelling and numerical solution of elastoacoustic vibrations with interface damping. International Journal for Numerical Methods in Engineering 1999; 46(10):1763-1779.

14. Legay A, Zilian A, Janssen C. A rheological interface model and its space-time finite element formulation for fluid-structure interaction. International Journal for Numerical Methods in Engineering 2011; 86(6):667-687.

15. Ihlenburg F, Babuska I. Finite element solution of the Helmholtz equation with high wave number. Part 1: the h-version of the FEM. Computers and Mathematics with Applications 1995; 38(9):9-37.

16. Bouillard Ph, Ihlenburg F. Error estimation and adaptivity for the finite element method in acoustics: 2D and 3D applications. Computer Methods in Applied Mechanics and Engineering 1999; 176(1-4):147-163.

17. Moès N, Dolbow J, Belytschko T. A finite element method for crack growth without remeshing. International Journal for Numerical Methods in Engineering 1999; 46(1):131-150.

18. Legay A. An extented finite element method approach for structural-acoustic problems involving immersed structures at arbitrary positions. International Journal for Numerical Methods in Engineering 2013; 93(4):376-399.

19. Legay A, Chessa J, Belytschko T. An Eulerian-Lagrangian method for fluid-structure interaction based on level sets. Computer Methods in Applied Mechanics and Engineering 2006; 195(17-18):2070-2087.

20. Zilian A, Legay A. The enriched space-time finite element method (EST) for simultaneous solution of fluid-structure interaction. International Journal for Numerical Methods in Engineering 2008; 75(3):305-334.

21. Gerstenberger A, Wall WA. An eXtended finite element method/Lagrange multiplier based approach for fluidstructure interaction. Computer Methods in Applied Mechanics and Engineering 2008; 197(19-20):1699-1714.

22. Duddu R, Bordas S, Chopp D, Moran B. A combined extended finite element and level set method for biofilm growth. International Journal for Numerical Methods in Engineering 2008; 74(5):848-870.

23. Chessa J, Belytschko T. An enriched finite element method and level sets for axisymmetric two-phase flow with surface tension. International Journal for Numerical Methods in Engineering 2003; 58(13):2041-2064. 
24. Sukumar N, Chopp DL, Moës N, Belytschko T. Modeling holes and inclusions by level sets in the extended finiteelement method. Computer Methods in Applied Mechanics and Engineering 2001; 190(46-47):6183-6200.

25. Legay A, Wang H, Belytschko T. Strong and weak arbitrary discontinuities in spectral finite elements. International Journal for Numerical Methods in Engineering 2005; 64(8):991-1008.

26. Wang H, Chessa J, Liu WK, Belytschko T. The immersed/fictitious element method for fluid-structure interaction: volumetric consistency, compressibility and thin members. International Journal for Numerical Methods in Engineering 2008; 74(1):32-55.

27. Wang X, Liu WK. Extended immersed boundary method using FEM and RKPM. Computer Methods in Applied Mechanics and Engineering 2003; 193(12-14):1305-1321.

28. Zhang L, Gerstenberger A, Wang X, Liu WK. Immersed finite element method. Computer Methods in Applied Mechanics and Engineering 2004; 193(21-22):2051-2067.

29. Laure P, Megally A, Coupez T. Collision strategy for the direct simulation of moving fibers in viscous fluid. International Conference on Computational Methods for Coupled, Problems in Science and Engineering Papadrakakis M, On̆ate E, Schrefler B (eds)., Barcelona, 2005; 1-13.

30. Rabczuk T, Gracie R, Song JH, Belytschko T. Immersed particle method for fluid-structure interaction. International Journal for Numerical Methods in Engineering 2010; 81(1):48-71.

31. Melenk JJ. The partition of unity finite element method: basic theory and applications. Computer Methods in Applied Mechanics and Engineering 1996; 139(1-4):289-314.

32. Chessa J, Wang H, Belytschko T. On the construction of blending elements for local partition of unity enriched finite elements. International Journal for Numerical Methods in Engineering 2003; 57(7):1015-1038.

33. Stricklin JA, Haisler W, Tisdale P, Gunderson R. A rapidly converging triangular plate element. AIAA Journal 1969; 7(1):180-181.

34. Dhatt G. An efficient triangular shell element. AIAA Journal 1970; 8(11):2100-2102.

35. Batoz J-L, Bathe K-J, Ho L-W. A study of three-node triangular plate bending elements. International Journal for Numerical Methods in Engineering 1980; 15(12):1771-1812.

36. Batoz JL, Dhatt G. Modélisation Des Structures Par éléments Finis, HERMES, vol. 3. Coques: Paris, 1992.

37. Craig RR, Bampton MCC. Coupling of substructures for dynamic analysis. AIAA Journal 1968; 6(7):1313-1319.

38. Geuzaine C, Remacle J-F. Gmsh: A 3-D finite element mesh generator with built-in pre- and post-processing facilities. International Journal for Numerical Methods in Engineering 2009; 79(11):1309-1331. 